\title{
Leptonic unitarity triangles: RGE running effects and $\mu-\tau$ reflection symmetry breaking
}

\author{
Jing-yu Zhu* \\ Institute of High Energy Physics, and School of Physical Sciences, \\ University of Chinese Academy of Sciences, Beijing 100049, China
}

(Received 13 October 2018; published 7 February 2019)

\begin{abstract}
There are six leptonic unitarity triangles (LUTs) defined by six orthogonality conditions of the threefamily lepton flavor mixing matrix in the complex plane. In the framework of the standard model or the minimal supersymmetric standard model, the evolutions of sides and inner angles of the six LUTs from a superhigh energy scale $\Lambda_{\mathrm{H}}$ to the electroweak scale $\Lambda_{\mathrm{EW}}$ due to the renormalization-group equation (RGE) running are derived in the integral form for both Dirac and Majorana neutrinos. Furthermore, the LUTs as an intuitively geometrical language are applied to the description of the RGE-induced $\mu-\tau$ reflection symmetry breaking analytically and numerically.
\end{abstract}

DOI: $10.1103 /$ PhysRevD.99.033003

\section{INTRODUCTION}

In the recent twenty years, a series of neutrino oscillation experiments have definitely proved that neutrinos have masses and lepton flavors mix with one another [1]. The latter can be described by the well-known PontecorvoMaki-Nakagawa-Sakata (PMNS) matrix $U[2,3]$, which connects three neutrino mass eigenstates $\left(\nu_{1}, \nu_{2}, \nu_{3}\right)$ and flavor eigenstates $\left(\nu_{e}, \nu_{\mu}, \nu_{\tau}\right)$ by

$$
\left(\begin{array}{c}
\nu_{e} \\
\nu_{\mu} \\
\nu_{\tau}
\end{array}\right)=\left(\begin{array}{ccc}
U_{e 1} & U_{e 2} & U_{e 3} \\
U_{\mu 1} & U_{\mu 2} & U_{\mu 3} \\
U_{\tau 1} & U_{\tau 2} & U_{\tau 3}
\end{array}\right)\left(\begin{array}{c}
\nu_{1} \\
\nu_{2} \\
\nu_{3}
\end{array}\right)
$$

in the basis where the mass eigenstates of three charged leptons are identical with their flavor eigenstates. According to the orthogonality of the rows and columns of $U$, one may define six leptonic unitarity triangles (LUTs) in the complex plane as a geometrical language to intuitively describe lepton flavor mixing and $C P$ violation [4]. The six triangles are

$$
\begin{aligned}
& \Delta_{e}: U_{\mu 1} U_{\tau 1}^{*}+U_{\mu 2} U_{\tau 2}^{*}+U_{\mu 3} U_{\tau 3}^{*}=0, \\
& \Delta_{\mu}: U_{\tau 1} U_{e 1}^{*}+U_{\tau 2} U_{e 2}^{*}+U_{\tau 3} U_{e 3}^{*}=0, \\
& \Delta_{\tau}: U_{e 1} U_{\mu 1}^{*}+U_{e 2} U_{\mu 2}^{*}+U_{e 3} U_{\mu 3}^{*}=0,
\end{aligned}
$$

\footnotetext{
"zhujingyu@ihep.ac.cn
}

Published by the American Physical Society under the terms of the Creative Commons Attribution 4.0 International license. Further distribution of this work must maintain attribution to the author(s) and the published article's title, journal citation, and DOI. Funded by SCOAP ${ }^{3}$. which are insensitive to the Majorana phases; and

$$
\begin{aligned}
& \Delta_{1}: U_{e 2} U_{e 3}^{*}+U_{\mu 2} U_{\mu 3}^{*}+U_{\tau 2} U_{\tau 3}^{*}=0, \\
& \Delta_{2}: U_{e 3} U_{e 1}^{*}+U_{\mu 3} U_{\mu 1}^{*}+U_{\tau 3} U_{\tau 1}^{*}=0, \\
& \Delta_{3}: U_{e 1} U_{e 2}^{*}+U_{\mu 1} U_{\mu 2}^{*}+U_{\tau 1} U_{\tau 2}^{*}=0,
\end{aligned}
$$

whose orientations are fixed by the Majorana phases ${ }^{1}$ [5]. The areas of these LUTs are all equal to $|\mathcal{J}| / 2$, where $\mathcal{J}$ means the Jarlskog invariant of $U$ describing leptonic $C P$ violation in neutrino oscillations and can be defined through

$$
\operatorname{Im}\left(U_{\alpha i} U_{\beta i}^{*} U_{\alpha j}^{*} U_{\beta j}\right)=\mathcal{J} \sum_{\gamma} \epsilon_{\alpha \beta \gamma} \sum_{k} \epsilon_{i j k} .
$$

The subscripts $(\alpha, \beta, \gamma)$ and $(i, j, k)$ in this paper always run over $(e, \mu, \tau)$ and $(1,2,3)$, respectively, if not otherwise specified. The six LUTs consist of eighteen vector sides in the complex plane shown in Eqs. (2) and (3) and nine inner angles which can be expressed as

$$
\phi_{\alpha i} \equiv \eta_{\phi} \arg \left[-\frac{U_{\beta j} U_{\gamma j}^{*}}{U_{\beta k} U_{\gamma k}^{*}}\right]=\eta_{\phi} \arg \left[-\frac{U_{\beta j} U_{\beta k}^{*}}{U_{\gamma j} U_{\gamma k}^{*}}\right] .
$$

\footnotetext{
${ }^{1}$ In the definitions of six LUTs in Eqs. (2) and (3), we do not consider the unphysical phases of $U$ for both Dirac and Majorana neutrinos. The orientations of LUTs correspond to possible rotations caused by unphysical or Majorana phases of $U$. So only the orientations of $\Delta_{i}$ of Majorana neutrinos have physical meaning.
} 
In Eq. (5), $\alpha, \beta$ and $\gamma$ run cyclically over $e, \mu$ and $\tau ; i, j$ and $k$ run cyclically over 1,2 and $3 ; \eta_{\phi}=1$ for $\mathcal{J}<0$ and $\eta_{\phi}=-1$ for $\mathcal{J}>0 .^{2}$

The language of LUTs has been discussed in a number of papers [6-12] since it was introduced into the lepton sector. These papers mainly focus on the following aspects:

(i) The reconstruction of LUTs through future precision neutrino oscillation and non-oscillation experiments will be a useful and intuitive geometric way to demonstrate $C P$ violation in the lepton sector, and this will be complementary to the direct measurements of $C P$ asymmetries [6,7]. Furthermore, testing whether the LUTs are close will provide tests of the unitarity of the PMNS matrix, which might be violated due to the existence of sterile neutrinos $[4,5,8]$.

(ii) One can directly use the sides and inner angles of the LUTs to describe neutrino-neutrino oscillations, neutrino-antineutrino oscillations and neutrino decays, where the inner angles of the LUTs have definite physical meaning $[9,10]$. The shapes of the LUTs can be reformed either by terrestrial matter effects, or by renormalization-group-equation (RGE) running effects, or by some other new physics effects, implying the corrections of such effects to lepton flavor mixing and $C P$ violation [11,12]. There are also discussions about the underlying phenomenological meaning of special shapes of the LUTs [10].

In Ref. [12], the RGE running behaviors of inner angles of the LUTs have been discussed in the differential form. In this paper, we aim to study how the sides and inner angles of the LUTs evolve in the integral form due to the RGE running from an arbitrary superhigh energy scale $\Lambda_{\mathrm{H}}$ to the electroweak scale $\Lambda_{\mathrm{EW}}$ in the framework of the standard model (SM) or the minimal supersymmetric standard model (MSSM). Both the cases of Dirac and Majorana neutrinos will be considered. We get the RGE-induced corrections to the LUTs by performing perturbative expansions. The final analytical results are independent of the parametrization of $U$. Assuming the $\mu-\tau$ reflection symmetry $[13,14]$ to be satisfied at a superhigh energy $\Lambda_{\mu \tau}$, the corresponding $\Delta_{i}$ should be isosceles triangles; $\Delta_{\mu}$ and $\Delta_{\tau}$ are congruent with each other. When running down to $\Lambda_{\mathrm{EW}}$, the $\mu-\tau$ reflection symmetry will be broken due the RGE running effects, leading to the deviations of the LUTs from their special shapes at $\Lambda_{\mu \tau}$. So the corrections to the LUTs from $\Lambda_{\mu \tau}$ to $\Lambda_{\mathrm{EW}}$ can be used to intuitively describe the corresponding RGE-induced $\mu-\tau$ reflection symmetry breaking, and thus

\footnotetext{
${ }^{2}$ We add $\eta_{\phi}$ to ensure that the inner angles are positive. In the relevant references, $\eta_{\phi}$ was often neglected.
}

it is meaningful to explore how the LUTs can be reformed analytically and numerically in this case.

The rest of this paper is organized as follows. In Sec. II, we derive the RGE-induced connections of the sides and inner angles of the LUTs between $\Lambda_{\mathrm{EW}}$ and $\Lambda_{\mathrm{H}}$ in the integral form in the framework of the SM or the MSSM, where both Dirac and Majorana neutrinos are considered. Section III is devoted to simplifying the analytically approximate results in Sec. II by assuming the $\mu-\tau$ reflection symmetry at $\Lambda_{\mu \tau}$. In Sec. IV, the RGE-induced deviations of the LUTs from the $\mu-\tau$ reflection symmetry limits will be studied numerically by scanning the complete parameter space, where the smallest neutrino mass and the MSSM parameter $\tan \beta$ at $\Lambda_{\mathrm{EW}}$ run in the reasonable ranges $[0,0.1] \mathrm{eV}$ and $[10,50]$, respectively, just as the way taken in Ref. [15]. The normal mass ordering (NMO) and inverted mass ordering (IMO) of Dirac or Majorana neutrinos will be considered. Finally, Sec. V is a summary of our main results.

\section{RGE RUNNING EFFECTS ON THE LUTS}

\section{A. The case of Dirac neutrinos}

Before a decisive measurement of the neutrinoless double-beta decay [16] verifies the Majorana nature of massive neutrinos, it is meaningful to consider the cases of both Dirac and Majorana neutrinos theoretically [17]. The evolution of the Dirac neutrino mass matrix from $\Lambda_{\mathrm{H}}$ to $\Lambda_{\mathrm{EW}}$ in the integral form can be written as [18]

$$
M_{\nu}^{\prime}=I_{0} T_{l} M_{\nu},
$$

where $M_{\nu}$ and $M_{\nu}^{\prime}$ are the Dirac neutrino mass matrix at $\Lambda_{\mathrm{H}}$ and $\Lambda_{\mathrm{EW}}$, respectively. Note that the notations with a prime superscript in the following text denote the parameters at $\Lambda_{\mathrm{EW}}$ and those without such a superscript stand for the corresponding parameters at $\Lambda_{\mathrm{H}}$ if not otherwise specified. Here we define $T_{l}=$ $\operatorname{Diag}\left\{I_{e}, I_{\mu}, I_{\tau}\right\}$ and

$$
\begin{aligned}
& I_{0}=\exp \left[\frac{1}{16 \pi^{2}} \int_{t^{\prime}}^{0} G \mathrm{~d} t\right], \\
& I_{\alpha}=\exp \left[-\frac{C_{l}}{16 \pi^{2}} \int_{t^{\prime}}^{0} y_{\alpha}^{2} \mathrm{~d} t\right],
\end{aligned}
$$

where $t \equiv \ln \left(\mu / \Lambda_{\mathrm{H}}\right)$ with $\mu$ being an arbitrary renormalization scale between $\Lambda_{\mathrm{EW}}$ and $\Lambda_{\mathrm{H}}$, and $t^{\prime} \equiv$ $\ln \left(\Lambda_{\mathrm{EW}} / \Lambda_{\mathrm{H}}\right)$. In the SM, one has $G=0.45 g_{1}^{2}+$ $2.25 g_{2}^{2}-3 y_{t}^{2}$ and $C_{l}=-1.5$; while in the MSSM, $G=$ $0.6 g_{1}^{2}+3 g_{2}^{2}-3 y_{t}^{2}$ and $C_{l}=1$, where $g_{1,2}$ denote the gauge couplings, and $y_{t}$ and $y_{\alpha}$ (for $\alpha=e, \mu, \tau$ ) stand respectively for the Yukawa couplings of the top quark 
and charged leptons. ${ }^{3}$ The Hermitian matrix $H_{\nu}=$ $M_{\nu} M_{\nu}^{\dagger}$ can be diagonalized by the unitary transformation $U^{\dagger} H_{\nu} U=\operatorname{Diag}\left\{m_{1}^{2}, m_{2}^{2}, m_{3}^{2}\right\}$ with $m_{i}$ being the neutrino masses. Similarly we have $H_{\nu}^{\prime}=M_{\nu}^{\prime} M_{\nu}^{\prime \dagger}$ and $U^{\prime \dagger} H_{\nu}^{\prime} U^{\prime}=\operatorname{Diag}\left\{m_{1}^{\prime 2}, m_{2}^{\prime 2}, m_{3}^{\prime 2}\right\}$ at $\Lambda_{\mathrm{EW}}$. According to Eq. (6), we directly write out

$$
\begin{aligned}
\left(H_{\nu}^{\prime}\right)_{\alpha \beta} & =\sum_{i} m_{i}^{\prime 2} U_{\alpha i}^{\prime} U_{\beta i}^{* *}=I_{0}^{2} I_{\alpha} I_{\beta}\left(H_{\nu}\right)_{\alpha \beta}, \\
\left(H_{\nu}^{\prime} H_{\nu}^{\prime \dagger}\right)_{\alpha \beta} & =\sum_{i} m_{i}^{\prime 4} U_{\alpha i}^{\prime} U_{\beta i}^{* *}=I_{0}^{4} I_{\alpha} I_{\beta} \sum_{\gamma} I_{\gamma}^{2}\left(H_{\nu}\right)_{\alpha \gamma}\left(H_{\nu}\right)_{\gamma \beta} .
\end{aligned}
$$

Then Eq. (8), together with the unitarity conditions of $U^{\prime}$, can yield a full set of linear equations of $U_{\alpha i}^{\prime} U_{\beta i}^{\prime *}$

$$
\begin{gathered}
\left(\begin{array}{ccc}
1 & 1 & 1 \\
m_{1}^{\prime 2} & m_{2}^{\prime 2} & m_{3}^{\prime 2} \\
m_{1}^{\prime 4} & m_{2}^{\prime 4} & m_{3}^{\prime 4}
\end{array}\right)\left(\begin{array}{c}
U_{\alpha 1}^{\prime} U_{\beta 1}^{\prime *} \\
U_{\alpha 2}^{\prime} U_{\beta 2}^{\prime *} \\
U_{\alpha 3}^{\prime} U_{\beta 3}^{\prime *}
\end{array}\right) \\
=\left(\begin{array}{c}
\delta_{\alpha \beta} \\
I_{0}^{2} I_{\alpha} I_{\beta}\left(H_{\nu}\right)_{\alpha \beta} \\
I_{0}^{4} I_{\alpha} I_{\beta} \sum_{\gamma} I_{\gamma}^{2}\left(H_{\nu}\right)_{\alpha \gamma}\left(H_{\nu}\right)_{\gamma \beta}
\end{array}\right)
\end{gathered}
$$

from which we can get exact expressions of $U_{\alpha i}^{\prime} U_{\beta i}^{*}$ at $\Lambda_{\mathrm{EW}}$. In addition, calculating the determinant of $H_{\nu}^{\prime}$ and the traces of $H_{\nu}^{\prime}$ and $H_{\nu}^{\prime} H_{\nu}^{\prime \dagger}$ from Eq. (8) leads to

$$
\begin{aligned}
m_{1}^{\prime 2} m_{2}^{\prime 2} m_{3}^{\prime 2} & =I_{0}^{6} I_{e}^{2} I_{\mu}^{2} I_{\tau}^{2} m_{1}^{2} m_{2}^{2} m_{3}^{2}, \\
m_{1}^{\prime 2}+m_{2}^{\prime 2}+m_{3}^{\prime 2} & =I_{0}^{2} \sum_{\alpha} I_{\alpha}^{2} \sum_{i} m_{i}^{2}\left|U_{\alpha i}\right|^{2}, \\
m_{1}^{\prime 4}+m_{2}^{\prime 4}+m_{3}^{\prime 4} & =I_{0}^{4} \sum_{\alpha} I_{\alpha}^{2} \sum_{\beta} I_{\beta}^{2}\left|\sum_{i} m_{i}^{2} U_{\alpha i} U_{\beta i}^{*}\right|^{2} .
\end{aligned}
$$

One can see $m_{i}^{\prime 2}$ (for $i=1,2,3$ and $m_{1}^{\prime 2} \neq m_{2}^{\prime 2} \neq m_{3}^{\prime 2}$ ) should be the solutions of the equation of $\lambda$ :

$$
\lambda^{3}-b \lambda^{2}+\frac{b^{2}-c}{2} \lambda-a=0,
$$

with $\quad a=m_{1}^{\prime 2} m_{2}^{\prime 2} m_{3}^{\prime 2}, \quad b=m_{1}^{\prime 2}+m_{2}^{\prime 2}+m_{3}^{\prime 2} \quad$ and $\quad c=$ $m_{1}^{\prime 4}+m_{2}^{\prime 4}+m_{3}^{\prime 4}$ coming from Eq. (10). The exact but complicated expressions of $m_{i}^{\prime 2}$ have been shown in Appendix. Here we perform some analytical approximations of Eq. (11) to see more clearly the dependence of $m_{i}^{\prime 2}$ on the parameters at $\Lambda_{\mathrm{H}}$. The taudominance approximation $T_{l} \simeq \operatorname{Diag}\{1,1,(1-\epsilon)\}$ will be taken due to the relationship $y_{e}^{2} \ll y_{\mu}^{2} \ll y_{\tau}^{2}$ with

$$
\epsilon=\frac{C_{l}}{16 \pi^{2}} \int_{t^{\prime}}^{0} y_{\tau}^{2} \mathrm{~d} t
$$

which is a small quantity, at most of order $\mathcal{O}(0.01)$ [15]. Expanding Eq. (11) in $\epsilon$ up to the first order gives rise to

$$
\begin{aligned}
& m_{1}^{\prime 2} \simeq I_{0}^{2} m_{1}^{2}\left(1-2 \epsilon\left|U_{\tau 1}\right|^{2}\right), \\
& m_{2}^{\prime 2} \simeq I_{0}^{2} m_{2}^{2}\left(1-2 \epsilon\left|U_{\tau 2}\right|^{2}\right), \\
& m_{3}^{\prime 2} \simeq I_{0}^{2} m_{3}^{2}\left(1-2 \epsilon\left|U_{\tau 3}\right|^{2}\right) .
\end{aligned}
$$

By inserting the tau-dominance approximation of $T_{l}$ and the above approximate results of $m_{i}^{\prime 2}$ into Eq. (9), and expanding it in $\epsilon$ up to the first order, we can arrive at the analytical approximations of $\left|U_{\alpha i}^{\prime}\right|^{2}$ at $\Lambda_{\mathrm{EW}}$ :

$$
\begin{aligned}
& \left|U_{e 1}^{\prime}\right|^{2} \simeq\left|U_{e 1}\right|^{2}-\frac{2 \epsilon}{\Delta_{21} \Delta_{31}}\left[\left(m_{2}^{2} m_{3}^{2}-m_{1}^{4}\right)\left|U_{e 1}\right|^{2}\left|U_{\tau 1}\right|^{2}+m_{1}^{2} \Delta_{32}\left(\left|U_{e 2}\right|^{2}\left|U_{\tau 2}\right|^{2}-\left|U_{e 3}\right|^{2}\left|U_{\tau 3}\right|^{2}\right)\right], \\
& \left|U_{e 2}^{\prime}\right|^{2} \simeq\left|U_{e 2}\right|^{2}+\frac{2 \epsilon}{\Delta_{21} \Delta_{32}}\left[\left(m_{1}^{2} m_{3}^{2}-m_{2}^{4}\right)\left|U_{e 2}\right|^{2}\left|U_{\tau 2}\right|^{2}+m_{2}^{2} \Delta_{31}\left(\left|U_{e 1}\right|^{2}\left|U_{\tau 1}\right|^{2}-\left|U_{e 3}\right|^{2}\left|U_{\tau 3}\right|^{2}\right)\right], \\
& \left|U_{e 3}^{\prime}\right|^{2} \simeq\left|U_{e 3}\right|^{2}-\frac{2 \epsilon}{\Delta_{31} \Delta_{32}}\left[\left(m_{1}^{2} m_{2}^{2}-m_{3}^{4}\right)\left|U_{e 3}\right|^{2}\left|U_{\tau 3}\right|^{2}+m_{3}^{2} \Delta_{21}\left(\left|U_{e 1}\right|^{2}\left|U_{\tau 1}\right|^{2}-\left|U_{e 2}\right|^{2}\left|U_{\tau 2}\right|^{2}\right)\right] ;
\end{aligned}
$$

and

$$
\begin{aligned}
& \left|U_{\mu 1}^{\prime}\right|^{2} \simeq\left|U_{\mu 1}\right|^{2}-\frac{2 \epsilon}{\Delta_{21} \Delta_{31}}\left[\left(m_{2}^{2} m_{3}^{2}-m_{1}^{4}\right)\left|U_{\mu 1}\right|^{2}\left|U_{\tau 1}\right|^{2}+m_{1}^{2} \Delta_{32}\left(\left|U_{\mu 2}\right|^{2}\left|U_{\tau 2}\right|^{2}-\left|U_{\mu 3}\right|^{2}\left|U_{\tau 3}\right|^{2}\right)\right], \\
& \left|U_{\mu 2}^{\prime}\right|^{2} \simeq\left|U_{\mu 2}\right|^{2}+\frac{2 \epsilon}{\Delta_{21} \Delta_{32}}\left[\left(m_{1}^{2} m_{3}^{2}-m_{2}^{4}\right)\left|U_{\mu 2}\right|^{2}\left|U_{\tau 2}\right|^{2}+m_{2}^{2} \Delta_{31}\left(\left|U_{\mu 1}\right|^{2}\left|U_{\tau 1}\right|^{2}-\left|U_{\mu 3}\right|^{2}\left|U_{\tau 3}\right|^{2}\right)\right], \\
& \left|U_{\mu 3}^{\prime}\right|^{2} \simeq\left|U_{\mu 3}\right|^{2}-\frac{2 \epsilon}{\Delta_{31} \Delta_{32}}\left[\left(m_{1}^{2} m_{2}^{2}-m_{3}^{4}\right)\left|U_{\mu 3}\right|^{2}\left|U_{\tau 3}\right|^{2}+m_{3}^{2} \Delta_{21}\left(\left|U_{\mu 1}\right|^{2}\left|U_{\tau 1}\right|^{2}-\left|U_{\mu 2}\right|^{2}\left|U_{\tau 2}\right|^{2}\right)\right] ;
\end{aligned}
$$

\footnotetext{
${ }^{3}$ Equations (6) and (25) can also apply to the two-Higgs-doublet models (2HDMs) [19] only by replacing the definitions of $G$ and $C_{l}$. For example, in type-II 2HDM, $C_{l}=-1.5$ and $G=0.45 g_{1}^{2}+2.25 g_{2}^{2}-y_{t}^{2}-3 y_{b}^{2}$ with $y_{b}$ being the bottom-quark Yukawa coupling [20].
} 
and

$$
\begin{aligned}
& \left|U_{\tau 1}^{\prime}\right|^{2} \simeq\left|U_{\tau 1}\right|^{2}+\frac{2 \epsilon\left|U_{\tau 1}\right|^{2}}{\Delta_{21} \Delta_{31}}\left[\left(m_{2}^{2} m_{3}^{2}-m_{1}^{4}\right)\left(1-\left|U_{\tau 1}\right|^{2}\right)+m_{1}^{2} \Delta_{32}\left(\left|U_{\tau 2}\right|^{2}-\left|U_{\tau 3}\right|^{2}\right)\right], \\
& \left|U_{\tau 2}^{\prime}\right|^{2} \simeq\left|U_{\tau 2}\right|^{2}-\frac{2 \epsilon\left|U_{\tau 2}\right|^{2}}{\Delta_{21} \Delta_{32}}\left[\left(m_{1}^{2} m_{3}^{2}-m_{2}^{4}\right)\left(1-\left|U_{\tau 2}\right|^{2}\right)+m_{2}^{2} \Delta_{31}\left(\left|U_{\tau 1}\right|^{2}-\left|U_{\tau 3}\right|^{2}\right)\right], \\
& \left|U_{\tau 3}^{\prime}\right|^{2} \simeq\left|U_{\tau 3}\right|^{2}+\frac{2 \epsilon\left|U_{\tau 3}\right|^{2}}{\Delta_{31} \Delta_{32}}\left[\left(m_{1}^{2} m_{2}^{2}-m_{3}^{4}\right)\left(1-\left|U_{\tau 3}\right|^{2}\right)+m_{3}^{2} \Delta_{21}\left(\left|U_{\tau 1}\right|^{2}-\left|U_{\tau 2}\right|^{2}\right)\right],
\end{aligned}
$$

where $\quad \Delta_{21}=m_{2}^{2}-m_{1}^{2}, \quad \Delta_{31}=m_{3}^{2}-m_{1}^{2}, \quad$ and $\quad \Delta_{32}=$ $m_{3}^{2}-m_{2}^{2}$. One can see that $\left|U_{\alpha i}^{\prime}\right|^{2}$ depend on $\left|U_{\tau i}\right|^{2}$ besides $\left|U_{\alpha i}\right|^{2}$ owing to the tau-dominance approximation of $T_{l}$, and there will be similar characteristics in the following results.
The analytical approximations of $\left|U_{\alpha i}^{\prime}\right|^{2}$ in Eqs. (14)-(16) satisfy $\sum_{i}\left|U_{\alpha i}^{\prime}\right|^{2}=\sum_{\alpha}\left|U_{\alpha i}^{\prime}\right|^{2}=1$. In the same way, we can obtain the analytical approximations of the vector sides of $\Delta_{\alpha}^{\prime}$ at $\Lambda_{\mathrm{EW}}$ :

$\begin{aligned} & U_{\mu 1}^{\prime} U_{\tau 1}^{*} \simeq U_{\mu 1} U_{\tau 1}^{*}+\frac{\epsilon}{\Delta_{21} \Delta_{31}}\left\{\left[\left(m_{2}^{2} m_{3}^{2}-m_{1}^{4}\right)\left(1-2\left|U_{\tau 1}\right|^{2}\right)-m_{1}^{2} \Delta_{32}\left(1-2\left|U_{\tau 2}\right|^{2}\right)\right] U_{\mu 1} U_{\tau 1}^{*}-2 m_{1}^{2} \Delta_{32}\left|U_{\tau 1}\right|^{2} U_{\mu 3} U_{\tau 3}^{*}\right\}, \\ & U_{\mu 2}^{\prime} U_{\tau 2}^{\prime *} \simeq U_{\mu 2} U_{\tau 2}^{*}-\frac{\epsilon}{\Delta_{21} \Delta_{32}}\left\{\left[\left(m_{1}^{2} m_{3}^{2}-m_{2}^{4}\right)\left(1-2\left|U_{\tau 2}\right|^{2}\right)-m_{2}^{2} \Delta_{31}\left(1-2\left|U_{\tau 1}\right|^{2}\right)\right] U_{\mu 2} U_{\tau 2}^{*}-2 m_{2}^{2} \Delta_{31}\left|U_{\tau 2}\right|^{2} U_{\mu 3} U_{\tau 3}^{*}\right\}, \\ & U_{\mu 3}^{\prime} U_{\tau 3}^{* *} \simeq U_{\mu 3} U_{\tau 3}^{*}+\frac{\epsilon}{\Delta_{31} \Delta_{32}}\left\{\left[\left(m_{1}^{2} m_{2}^{2}-m_{3}^{4}\right)\left(1-2\left|U_{\tau 3}\right|^{2}\right)+m_{3}^{2} \Delta_{21}\left(1-2\left|U_{\tau 2}\right|^{2}\right)\right] U_{\mu 3} U_{\tau 3}^{*}+2 m_{3}^{2} \Delta_{21}\left|U_{\tau 3}\right|^{2} U_{\mu 1} U_{\tau 1}^{*}\right\},\end{aligned}$

for $\Delta_{e}^{\prime}$; and

$U_{\tau 1}^{\prime} U_{e 1}^{\prime *} \simeq U_{\tau 1} U_{e 1}^{*}+\frac{\epsilon}{\Delta_{21} \Delta_{31}}\left\{\left[\left(m_{2}^{2} m_{3}^{2}-m_{1}^{4}\right)\left(1-2\left|U_{\tau 1}\right|^{2}\right)-m_{1}^{2} \Delta_{32}\left(1-2\left|U_{\tau 2}\right|^{2}\right)\right] U_{\tau 1} U_{e 1}^{*}-2 m_{1}^{2} \Delta_{32}\left|U_{\tau 1}\right|^{2} U_{\tau 3} U_{e 3}^{*}\right\}$,

$U_{\tau 2}^{\prime} U_{e 2}^{*} \simeq U_{\tau 2} U_{e 2}^{*}-\frac{\epsilon}{\Delta_{21} \Delta_{32}}\left\{\left[\left(m_{1}^{2} m_{3}^{2}-m_{2}^{4}\right)\left(1-2\left|U_{\tau 2}\right|^{2}\right)-m_{2}^{2} \Delta_{31}\left(1-2\left|U_{\tau 1}\right|^{2}\right)\right] U_{\tau 2} U_{e 2}^{*}-2 m_{2}^{2} \Delta_{31}\left|U_{\tau 2}\right|^{2} U_{\tau 3} U_{e 3}^{*}\right\}$,

$U_{\tau 3}^{\prime} U_{e 3}^{\prime *} \simeq U_{\tau 3} U_{e 3}^{*}+\frac{\epsilon}{\Delta_{31} \Delta_{32}}\left\{\left[\left(m_{1}^{2} m_{2}^{2}-m_{3}^{4}\right)\left(1-2\left|U_{\tau 3}\right|^{2}\right)+m_{3}^{2} \Delta_{21}\left(1-2\left|U_{\tau 2}\right|^{2}\right)\right] U_{\tau 3} U_{e 3}^{*}+2 m_{3}^{2} \Delta_{21}\left|U_{\tau 3}\right|^{2} U_{\tau 1} U_{e 1}^{*}\right\}$,

for $\Delta_{\mu}^{\prime}$; and

$$
\begin{aligned}
& U_{e 1}^{\prime} U_{\mu 1}^{*} \simeq U_{e 1} U_{\mu 1}^{*}-\frac{2 \epsilon}{\Delta_{21} \Delta_{31}}\left\{\left[\left(m_{2}^{2} m_{3}^{2}-m_{1}^{4}\right)\left|U_{\tau 1}\right|^{2}-m_{1}^{2} \Delta_{32}\left|U_{\tau 2}\right|^{2}\right] U_{e 1} U_{\mu 1}^{*}-m_{1}^{2} \Delta_{32}\left(1-\left|U_{\tau 1}\right|^{2}\right) U_{e 3} U_{\mu 3}^{*}\right\}, \\
& U_{e 2}^{\prime} U_{\mu 2}^{\prime *} \simeq U_{e 2} U_{\mu 2}^{*}+\frac{2 \epsilon}{\Delta_{21} \Delta_{32}}\left\{\left[\left(m_{1}^{2} m_{3}^{2}-m_{2}^{4}\right)\left|U_{\tau 2}\right|^{2}-m_{2}^{2} \Delta_{31}\left|U_{\tau 1}\right|^{2}\right] U_{e 2} U_{\mu 2}^{*}-m_{2}^{2} \Delta_{31}\left(1-\left|U_{\tau 2}\right|^{2}\right) U_{e 3} U_{\mu 3}^{*}\right\}, \\
& U_{e 3}^{\prime} U_{\mu 3}^{\prime *} \simeq U_{e 3} U_{\mu 3}^{*}-\frac{2 \epsilon}{\Delta_{31} \Delta_{32}}\left\{\left[\left(m_{1}^{2} m_{2}^{2}-m_{3}^{4}\right)\left|U_{\tau 3}\right|^{2}+m_{3}^{2} \Delta_{21}\left|U_{\tau 2}\right|^{2}\right] U_{e 3} U_{\mu 3}^{*}+m_{3}^{2} \Delta_{21}\left(1-\left|U_{\tau 3}\right|^{2}\right) U_{e 1} U_{\mu 1}^{*}\right\},
\end{aligned}
$$

for $\Delta_{\tau}^{\prime}$, where $\sum_{i} U_{\alpha i}^{\prime} U_{\beta i}^{\prime *}=0$ holds for $(\alpha, \beta)=(e, \mu),(\mu, \tau),(\tau, e)$. Considering the fact that the lengths of three sides of each $\Delta_{i}^{\prime}$ can be derived from Eqs. (14)-(16), one can see that the six LUTs $\left(\Delta_{\alpha}^{\prime}\right.$ and $\left.\Delta_{i}^{\prime}\right)$ of the Dirac neutrinos at $\Lambda_{\mathrm{EW}}$ can be approximately fixed from the above results. Furthermore, we can get the approximate Jarlskog invariant $\mathcal{J}^{\prime}$ of the Dirac neutrinos at $\Lambda_{\mathrm{EW}}$ from anyone of Eqs. (17)-(19). The result is

$$
\mathcal{J}^{\prime} \simeq \mathcal{J}-\frac{2 \epsilon \mathcal{J}}{\Delta_{21} \Delta_{31} \Delta_{32}}\left[m_{1}^{2}\left(m_{2}^{4}+m_{3}^{4}\right)\left(\left|U_{\tau 3}\right|^{2}-\left|U_{\tau 2}\right|^{2}\right)+m_{2}^{2}\left(m_{1}^{4}+m_{3}^{4}\right)\left(\left|U_{\tau 1}\right|^{2}-\left|U_{\tau 3}\right|^{2}\right)+m_{3}^{2}\left(m_{1}^{4}+m_{2}^{4}\right)\left(\left|U_{\tau 2}\right|^{2}-\left|U_{\tau 1}\right|^{2}\right)\right] .
$$

With the help of Eqs. (4) and (5), one can define

$$
\cot \phi_{\alpha i} \equiv \frac{\eta_{\phi}}{\mathcal{J}} \operatorname{Re}\left(U_{\beta j} U_{\gamma j}^{*} U_{\beta k}^{*} U_{\gamma k}\right), \quad \cot \phi_{\alpha i}^{\prime} \equiv \frac{\eta_{\phi}}{\mathcal{J}^{\prime}} \operatorname{Re}\left(U_{\beta j}^{\prime} U_{\gamma j}^{\prime *} U_{\beta k}^{*} U_{\gamma k}^{\prime}\right),
$$


where $(\alpha, \beta, \gamma)$ and $(i, j, k)$ run cyclically over $(e, \mu, \tau)$ and $(1,2,3)$, respectively. We then calculate the evolutions of the nine inner angles of LUTs for the Dirac neutrinos from $\Lambda_{\mathrm{H}}$ to $\Lambda_{\mathrm{EW}}$ by combining Eqs. (17)-(19) and (21), and obtain

$$
\begin{aligned}
& \cot \phi_{e 1}^{\prime} \simeq \cot \phi_{e 1}+\eta_{\phi} \frac{2 \epsilon\left|U_{\tau 2}\right|^{2}\left|U_{\tau 3}\right|^{2}}{\mathcal{J} \Delta_{21} \Delta_{31} \Delta_{32}}\left(m_{2}^{2} \Delta_{31}^{2}\left|U_{\mu 3}\right|^{2}-m_{3}^{2} \Delta_{21}^{2}\left|U_{\mu 2}\right|^{2}\right), \\
& \cot \phi_{e 2}^{\prime} \simeq \cot \phi_{e 2}+\eta_{\phi} \frac{2 \epsilon\left|U_{\tau 1}\right|^{2}\left|U_{\tau 3}\right|^{2}}{\mathcal{J} \Delta_{21} \Delta_{31} \Delta_{32}}\left(m_{3}^{2} \Delta_{21}^{2}\left|U_{\mu 1}\right|^{2}-m_{1}^{2} \Delta_{32}^{2}\left|U_{\mu 3}\right|^{2}\right), \\
& \cot \phi_{e 3}^{\prime} \simeq \cot \phi_{e 3}+\eta_{\phi} \frac{2 \epsilon\left|U_{\tau 1}\right|^{2}\left|U_{\tau 2}\right|^{2}}{\mathcal{J} \Delta_{21} \Delta_{31} \Delta_{32}}\left(m_{1}^{2} \Delta_{32}^{2}\left|U_{\mu 2}\right|^{2}-m_{2}^{2} \Delta_{31}^{2}\left|U_{\mu 1}\right|^{2}\right) ;
\end{aligned}
$$

and

$$
\begin{aligned}
& \cot \phi_{\mu 1}^{\prime} \simeq \cot \phi_{\mu 1}+\eta_{\phi} \frac{2 \epsilon\left|U_{\tau 2}\right|^{2}\left|U_{\tau 3}\right|^{2}}{\mathcal{J} \Delta_{21} \Delta_{31} \Delta_{32}}\left(m_{2}^{2} \Delta_{31}^{2}\left|U_{e 3}\right|^{2}-m_{3}^{2} \Delta_{21}^{2}\left|U_{e 2}\right|^{2}\right), \\
& \cot \phi_{\mu 2}^{\prime} \simeq \cot \phi_{\mu 2}+\eta_{\phi} \frac{2 \epsilon\left|U_{\tau 1}\right|^{2}\left|U_{\tau 3}\right|^{2}}{\mathcal{J} \Delta_{21} \Delta_{31} \Delta_{32}}\left(m_{3}^{2} \Delta_{21}^{2}\left|U_{e 1}\right|^{2}-m_{1}^{2} \Delta_{32}^{2}\left|U_{e 3}\right|^{2}\right), \\
& \cot \phi_{\mu 3}^{\prime} \simeq \cot \phi_{\mu 3}+\eta_{\phi} \frac{2 \epsilon\left|U_{\tau 1}\right|^{2}\left|U_{\tau 2}\right|^{2}}{\mathcal{J} \Delta_{21} \Delta_{31} \Delta_{32}}\left(m_{1}^{2} \Delta_{32}^{2}\left|U_{e 2}\right|^{2}-m_{2}^{2} \Delta_{31}^{2}\left|U_{e 1}\right|^{2}\right) ;
\end{aligned}
$$

and

$$
\begin{aligned}
& \cot \phi_{\tau 1}^{\prime} \simeq \cot \phi_{\tau 1}+\frac{2 \eta_{\phi} \epsilon}{\mathcal{J} \Delta_{21} \Delta_{31} \Delta_{32}}\left[m_{3}^{2} \Delta_{21}^{2}\left|U_{e 2} U_{\mu 2}^{*}\right|^{2}\left(1-\left|U_{\tau 3}\right|^{2}\right)-m_{2}^{2} \Delta_{31}^{2}\left|U_{e 3} U_{\mu 3}^{*}\right|^{2}\left(1-\left|U_{\tau 2}\right|^{2}\right)\right], \\
& \cot \phi_{\tau 2}^{\prime} \simeq \cot \phi_{\tau 2}+\frac{2 \eta_{\phi} \epsilon}{\mathcal{J} \Delta_{21} \Delta_{31} \Delta_{32}}\left[m_{1}^{2} \Delta_{32}^{2}\left|U_{e 3} U_{\mu 3}^{*}\right|^{2}\left(1-\left|U_{\tau 1}\right|^{2}\right)-m_{3}^{2} \Delta_{21}^{2}\left|U_{e 1} U_{\mu 1}^{*}\right|^{2}\left(1-\left|U_{\tau 3}\right|^{2}\right)\right], \\
& \cot \phi_{\tau 3}^{\prime} \simeq \cot \phi_{\tau 3}+\frac{2 \eta_{\phi} \epsilon}{\mathcal{J} \Delta_{21} \Delta_{31} \Delta_{32}}\left[m_{2}^{2} \Delta_{31}^{2}\left|U_{e 1} U_{\mu 1}^{*}\right|^{2}\left(1-\left|U_{\tau 2}\right|^{2}\right)-m_{1}^{2} \Delta_{32}^{2}\left|U_{e 2} U_{\mu 2}^{*}\right|^{2}\left(1-\left|U_{\tau 1}\right|^{2}\right)\right] .
\end{aligned}
$$

\section{B. The case of Majorana neutrinos}

When considering the Majorana neutrinos, one can naturally explain their small masses through the seesaw mechanisms [21]. The evolution of the Majorana neutrino mass matrix from $\Lambda_{\mathrm{H}}$ to $\Lambda_{\mathrm{EW}}$ in the integral form can be written as $[4,22]$

$$
M_{\nu}^{\prime}=I_{0}^{2} T_{l} M_{\nu} T_{l} .
$$

Note that $M_{\nu}$ and $M_{\nu}^{\prime}$ represent the Majorana neutrino mass matrices at $\Lambda_{\mathrm{H}}$ and $\Lambda_{\mathrm{EW}}$, respectively. $I_{0}$ and $T_{l}$ have been defined below Eq. (6) and in Eq. (7). We can also derive the direct connections of the LUTs between the two energy scales $\Lambda_{\mathrm{H}}$ and $\Lambda_{\mathrm{EW}}$ as in the Dirac case. Let us repeat the similar calculations below for comparison. We first diagonalize $M_{\nu}$ and $M_{\nu}^{\prime}$ through $U^{\dagger} M_{\nu} U^{*}=\operatorname{Diag}\left\{m_{1}^{2}, m_{2}^{2}, m_{3}^{2}\right\}$ and $\quad U^{\prime \dagger} M_{\nu}^{\prime} U^{\prime *}=\operatorname{Diag}\left\{m_{1}^{\prime 2}, m_{2}^{\prime 2}, m_{3}^{\prime 2}\right\}$. According to Eq. (25), the Hermitian matrices $H_{\nu}^{\prime} \equiv M_{\nu} M_{\nu}^{\dagger}$ and $H_{\nu}^{\prime} H_{\nu}^{\prime \dagger}$ for the case of Majorana neutrinos can be expressed as

$$
\begin{aligned}
\left(H_{\nu}^{\prime}\right)_{\alpha \beta} & =\sum_{i} m_{i}^{\prime 2} U_{\alpha i}^{\prime} U_{\beta i}^{\prime *}=I_{0}^{4} I_{\alpha} I_{\beta} Q_{\alpha \beta}, \\
\left(H_{\nu}^{\prime} H_{\nu}^{\prime \dagger}\right)_{\alpha \beta} & =\sum_{i} m_{i}^{\prime 4} U_{\alpha i}^{\prime} U_{\beta i}^{\prime *}=I_{0}^{8} I_{\alpha} I_{\beta} \sum_{\gamma} I_{\gamma}^{2} Q_{\alpha \gamma} Q_{\gamma \beta},
\end{aligned}
$$

where we have defined $Q \equiv M_{\nu} T_{l}^{2} M_{\nu}^{\dagger}$ for simplicity. With the help of Eq. (26) and the unitarity conditions of $U^{\prime}$, we can get

$$
\left(\begin{array}{ccc}
1 & 1 & 1 \\
m_{1}^{\prime 2} & m_{2}^{\prime 2} & m_{3}^{\prime 2} \\
m_{1}^{\prime 4} & m_{2}^{\prime 4} & m_{3}^{\prime 4}
\end{array}\right)\left(\begin{array}{c}
U_{\alpha 1}^{\prime} U_{\beta 1}^{\prime *} \\
U_{\alpha 2}^{\prime} U_{\beta 2}^{\prime *} \\
U_{\alpha 3}^{\prime} U_{\beta 3}^{\prime *}
\end{array}\right)=\left(\begin{array}{c}
\delta_{\alpha \beta} \\
I_{0}^{4} I_{\alpha} I_{\beta} Q_{\alpha \beta} \\
I_{0}^{8} I_{\alpha} I_{\beta} \sum_{\gamma} I_{\gamma}^{2} Q_{\alpha \gamma} Q_{\gamma \beta}
\end{array}\right) .
$$

Moreover, the determinant of $H_{\nu}^{\prime}$ together with the traces of $H_{\nu}^{\prime}$ and $H_{\nu}^{\prime} H_{\nu}^{\prime \dagger}$ leads to

$$
\begin{aligned}
m_{1}^{\prime 2} m_{2}^{\prime 2} m_{3}^{\prime 2} & =I_{0}^{12} I_{e}^{4} I_{\mu}^{4} I_{\tau}^{4} m_{1}^{2} m_{2}^{2} m_{3}^{2}, \\
m_{1}^{\prime 2}+m_{2}^{\prime 2}+m_{3}^{\prime 2} & =I_{0}^{4} \sum_{\alpha} I_{\alpha}^{2} \sum_{\beta} I_{\beta}^{2}\left|\left(M_{\nu}\right)_{\alpha \beta}\right|^{2}, \\
m_{1}^{\prime 4}+m_{2}^{\prime 4}+m_{3}^{\prime 4} & =I_{0}^{8} \sum_{\alpha} I_{\alpha}^{2} \sum_{\beta} I_{\beta}^{2}\left|\sum_{\gamma} I_{\gamma}^{2}\left(M_{\nu}\right)_{\alpha \gamma}\left(M_{\nu}\right)_{\beta \gamma}^{*}\right|^{2},
\end{aligned}
$$

where $\left(M_{\nu}\right)_{\alpha \beta}=\sum_{i} m_{i} U_{\alpha i} U_{\beta i}$. By solving the equation

$$
\lambda^{3}-b \lambda^{2}+\frac{b^{2}-c}{2} \lambda-a=0,
$$


with $a=m_{1}^{\prime 2} m_{2}^{\prime 2} m_{3}^{\prime 2}, b=m_{1}^{\prime 2}+m_{2}^{\prime 2}+m_{3}^{\prime 2}$ and $c=m_{1}^{\prime 4}+m_{2}^{\prime 4}+m_{3}^{\prime 4}$ coming from Eq. (28), the exact expressions of $m_{i}^{\prime 2}$ for Majorana neutrinos can be derived. One may refer to Appendix for their specific expressions. Here we calculate $m_{i}^{\prime 2}$ approximately by expanding Eq. (29) in $\epsilon$, and arrive at

$$
m_{1}^{\prime 2} \simeq I_{0}^{4} m_{1}^{2}\left(1-4 \epsilon\left|U_{\tau 1}\right|^{2}\right), \quad m_{2}^{\prime 2} \simeq I_{0}^{4} m_{2}^{2}\left(1-4 \epsilon\left|U_{\tau 2}\right|^{2}\right), \quad m_{3}^{\prime 2} \simeq I_{0}^{4} m_{3}^{2}\left(1-4 \epsilon\left|U_{\tau 3}\right|^{2}\right) .
$$

By inserting the tau-dominance approximation of $T_{l}$ and Eq. (30) into Eq. (27), and expanding it in $\epsilon$ up to the first order, we can get the analytical approximations of $\left|U_{\alpha i}^{\prime}\right|^{2}$ at $\Lambda_{\mathrm{EW}}$ :

$$
\begin{aligned}
\left|U_{e 1}^{\prime}\right|^{2} \simeq & \left|U_{e 1}\right|^{2}-\frac{2 \epsilon}{\Delta_{21} \Delta_{31}}\left[\left(m_{2}^{2} m_{3}^{2}-m_{1}^{4}\right)\left|U_{e 1}\right|^{2}\left|U_{\tau 1}\right|^{2}+m_{1}^{2} \Delta_{32}\left(\left|U_{e 2}\right|^{2}\left|U_{\tau 2}\right|^{2}-\left|U_{e 3}\right|^{2}\left|U_{\tau 3}\right|^{2}\right)\right. \\
& \left.-2 m_{1} m_{2} \Delta_{31} \mathbb{R}_{e \tau}^{12}-2 m_{1} m_{3} \Delta_{21} \mathbb{R}_{e \tau}^{13}\right] \\
\left|U_{e 2}^{\prime}\right|^{2} \simeq & \left|U_{e 2}\right|^{2}+\frac{2 \epsilon}{\Delta_{21} \Delta_{32}}\left[\left(m_{1}^{2} m_{3}^{2}-m_{2}^{4}\right)\left|U_{e 2}\right|^{2}\left|U_{\tau 2}\right|^{2}+m_{2}^{2} \Delta_{31}\left(\left|U_{e 1}\right|^{2}\left|U_{\tau 1}\right|^{2}-\left|U_{e 3}\right|^{2}\left|U_{\tau 3}\right|^{2}\right)\right. \\
& \left.-2 m_{1} m_{2} \Delta_{32} \mathbb{R}_{e \tau}^{12}+2 m_{2} m_{3} \Delta_{21} \mathbb{R}_{e \tau}^{23}\right] \\
\left|U_{e 3}^{\prime}\right|^{2} \simeq & \left|U_{e 3}\right|^{2}-\frac{2 \epsilon}{\Delta_{31} \Delta_{32}}\left[\left(m_{1}^{2} m_{2}^{2}-m_{3}^{4}\right)\left|U_{e 3}\right|^{2}\left|U_{\tau 3}\right|^{2}-m_{3}^{2} \Delta_{21}\left(\left|U_{e 2}\right|^{2}\left|U_{\tau 2}\right|^{2}-\left|U_{e 1}\right|^{2}\left|U_{\tau 1}\right|^{2}\right)\right. \\
& \left.+2 m_{1} m_{3} \Delta_{32} \mathbb{R}_{e \tau}^{13}+2 m_{2} m_{3} \Delta_{31} \mathbb{R}_{e \tau}^{23}\right]
\end{aligned}
$$

and

$$
\begin{aligned}
\left|U_{\mu 1}^{\prime}\right|^{2} \simeq & \left|U_{\mu 1}\right|^{2}-\frac{2 \epsilon}{\Delta_{21} \Delta_{31}}\left[\left(m_{2}^{2} m_{3}^{2}-m_{1}^{4}\right)\left|U_{\mu 1}\right|^{2}\left|U_{\tau 1}\right|^{2}+m_{1}^{2} \Delta_{32}\left(\left|U_{\mu 2}\right|^{2}\left|U_{\tau 2}\right|^{2}-\left|U_{\mu 3}\right|^{2}\left|U_{\tau 3}\right|^{2}\right)\right. \\
& \left.-2 m_{1} m_{2} \Delta_{31} \mathbb{R}_{\mu \tau}^{12}-2 m_{1} m_{3} \Delta_{21} \mathbb{R}_{\mu \tau}^{13}\right] \\
\left|U_{\mu 2}^{\prime}\right|^{2} \simeq & \left|U_{\mu 2}\right|^{2}+\frac{2 \epsilon}{\Delta_{21} \Delta_{32}}\left[\left(m_{1}^{2} m_{3}^{2}-m_{2}^{4}\right)\left|U_{\mu 2}\right|^{2}\left|U_{\tau 2}\right|^{2}+m_{2}^{2} \Delta_{31}\left(\left|U_{\mu 1}\right|^{2}\left|U_{\tau 1}\right|^{2}-\left|U_{\mu 3}\right|^{2}\left|U_{\tau 3}\right|^{2}\right)\right. \\
& \left.-2 m_{1} m_{2} \Delta_{32} \mathbb{R}_{\mu \tau}^{12}+2 m_{2} m_{3} \Delta_{21} \mathbb{R}_{\mu \tau}^{23}\right] \\
\left|U_{\mu 3}^{\prime}\right|^{2} \simeq & \left|U_{\mu 3}\right|^{2}-\frac{2 \epsilon}{\Delta_{31} \Delta_{32}}\left[\left(m_{1}^{2} m_{2}^{2}-m_{3}^{4}\right)\left|U_{\mu 3}\right|^{2}\left|U_{\tau 3}\right|^{2}-m_{3}^{2} \Delta_{21}\left(\left|U_{\mu 2}\right|^{2}\left|U_{\tau 2}\right|^{2}-\left|U_{\mu 1}\right|^{2}\left|U_{\tau 1}\right|^{2}\right)\right. \\
& \left.+2 m_{1} m_{3} \Delta_{32} \mathbb{R}_{\mu \tau}^{13}+2 m_{2} m_{3} \Delta_{31} \mathbb{R}_{\mu \tau}^{23}\right]
\end{aligned}
$$

and

$$
\begin{aligned}
\left|U_{\tau 1}^{\prime}\right|^{2} \simeq & \left|U_{\tau 1}\right|^{2}+\frac{2 \epsilon}{\Delta_{21} \Delta_{31}}\left[\left(m_{2}^{2} m_{3}^{2}-m_{1}^{4}\right)\left|U_{\tau 1}\right|^{2}\left(1-\left|U_{\tau 1}\right|^{2}\right)+m_{1}^{2} \Delta_{32}\left|U_{\tau 1}\right|^{2}\left(\left|U_{\tau 2}\right|^{2}-\left|U_{\tau 3}\right|^{2}\right)\right. \\
& \left.+2 m_{1} m_{2} \Delta_{31} \mathbb{R}_{\tau \tau}^{12}+2 m_{1} m_{3} \Delta_{21} \mathbb{R}_{\tau \tau}^{13}\right], \\
\left|U_{\tau 2}^{\prime}\right|^{2} \simeq & \left|U_{\tau 2}\right|^{2}-\frac{2 \epsilon}{\Delta_{21} \Delta_{32}}\left[\left(m_{1}^{2} m_{3}^{2}-m_{2}^{4}\right)\left|U_{\tau 2}\right|^{2}\left(1-\left|U_{\tau 2}\right|^{2}\right)+m_{2}^{2} \Delta_{31}\left|U_{\tau 2}\right|^{2}\left(\left|U_{\tau 1}\right|^{2}-\left|U_{\tau 3}\right|^{2}\right)\right. \\
& \left.+2 m_{1} m_{2} \Delta_{32} \mathbb{R}_{\tau \tau}^{12}-2 m_{2} m_{3} \Delta_{21} \mathbb{R}_{\tau \tau}^{23}\right], \\
\left|U_{\tau 3}^{\prime}\right|^{2} \simeq & \left|U_{\tau 3}\right|^{2}+\frac{2 \epsilon}{\Delta_{31} \Delta_{32}}\left[\left(m_{1}^{2} m_{2}^{2}-m_{3}^{4}\right)\left|U_{\tau 3}\right|^{2}\left(1-\left|U_{\tau 3}\right|^{2}\right)-m_{3}^{2} \Delta_{21}\left|U_{\tau 3}\right|^{2}\left(\left|U_{\tau 2}\right|^{2}-\left|U_{\tau 1}\right|^{2}\right)\right. \\
& \left.-2 m_{1} m_{3} \Delta_{32} \mathbb{R}_{\tau \tau}^{13}-2 m_{2} m_{3} \Delta_{31} \mathbb{R}_{\tau \tau}^{23}\right],
\end{aligned}
$$

with $\mathbb{R}_{\alpha \beta}^{i j}$ denoting the real parts of $U_{\alpha i} U_{\alpha j}^{*} U_{\beta i} U_{\beta j}^{*}$. The vector sides of $\Delta_{\alpha}^{\prime}$ at $\Lambda_{\mathrm{EW}}$ turn out to be: 


$$
\begin{aligned}
U_{\mu 1}^{\prime} U_{\tau 1}^{\prime *} \simeq & U_{\mu 1} U_{\tau 1}^{*}+\frac{\epsilon}{\Delta_{21} \Delta_{31}}\left\{\left[\left(m_{2}^{2} m_{3}^{2}-m_{1}^{4}\right)\left(1-2\left|U_{\tau 1}\right|^{2}\right)-m_{1}^{2} \Delta_{32}\left(1-2\left|U_{\tau 2}\right|^{2}\right)\right] U_{\mu 1} U_{\tau 1}^{*}\right. \\
& -2 m_{1}^{2} \Delta_{32}\left|U_{\tau 1}\right|^{2} U_{\mu 3} U_{\tau 3}^{*}+2 m_{1} m_{2} \Delta_{31}\left(U_{\mu 1} U_{\tau 1} U_{\tau 2}^{* 2}+U_{\mu 2} U_{\tau 2} U_{\tau 1}^{* 2}\right) \\
& \left.+2 m_{1} m_{3} \Delta_{21}\left(U_{\mu 1} U_{\tau 1} U_{\tau 3}^{* 2}+U_{\mu 3} U_{\tau 3} U_{\tau 1}^{* 2}\right)\right\}, \\
U_{\mu 2}^{\prime} U_{\tau 2}^{\prime *} \simeq & U_{\mu 2} U_{\tau 2}^{*}-\frac{\epsilon}{\Delta_{21} \Delta_{32}}\left\{\left[\left(m_{1}^{2} m_{3}^{2}-m_{2}^{4}\right)\left(1-2\left|U_{\tau 2}\right|^{2}\right)-m_{2}^{2} \Delta_{31}\left(1-2\left|U_{\tau 1}\right|^{2}\right)\right] U_{\mu 2} U_{\tau 2}^{*}\right. \\
& -2 m_{2}^{2} \Delta_{31}\left|U_{\tau 2}\right|^{2} U_{\mu 3} U_{\tau 3}^{*}+2 m_{1} m_{2} \Delta_{32}\left(U_{\mu 1} U_{\tau 1} U_{\tau 2}^{* 2}+U_{\mu 2} U_{\tau 2} U_{\tau 1}^{* 2}\right) \\
& \left.-2 m_{2} m_{3} \Delta_{21}\left(U_{\mu 2} U_{\tau 2} U_{\tau 3}^{* 2}+U_{\mu 3} U_{\tau 3} U_{\tau 2}^{* 2}\right)\right\}, \\
U_{\mu 3}^{\prime} U_{\tau 3}^{* *} \simeq & U_{\mu 3} U_{\tau 3}^{*}+\frac{\epsilon}{\Delta_{31} \Delta_{32}}\left\{\left[\left(m_{1}^{2} m_{2}^{2}-m_{3}^{4}\right)\left(1-2\left|U_{\tau 3}\right|^{2}\right)+m_{3}^{2} \Delta_{21}\left(1-2\left|U_{\tau 2}\right|^{2}\right)\right] U_{\mu 3} U_{\tau 3}^{*}\right. \\
& +2 m_{3}^{2} \Delta_{21}\left|U_{\tau 3}\right|^{2} U_{\mu 1} U_{\tau 1}^{*}-2 m_{2} m_{3} \Delta_{31}\left(U_{\mu 2} U_{\tau 2} U_{\tau 3}^{* 2}+U_{\mu 3} U_{\tau 3} U_{\tau 2}^{* 2}\right) \\
& \left.-2 m_{1} m_{3} \Delta_{32}\left(U_{\mu 1} U_{\tau 1} U_{\tau 3}^{* 2}+U_{\mu 3} U_{\tau 3} U_{\tau 1}^{* 2}\right)\right\},
\end{aligned}
$$

for $\Delta_{e}^{\prime}$; and

$$
\begin{aligned}
U_{\tau 1}^{\prime} U_{e 1}^{*} \simeq & U_{\tau 1} U_{e 1}^{*}+\frac{\epsilon}{\Delta_{21} \Delta_{31}}\left\{\left[\left(m_{2}^{2} m_{3}^{2}-m_{1}^{4}\right)\left(1-2\left|U_{\tau 1}\right|^{2}\right)-m_{1}^{2} \Delta_{32}\left(1-2\left|U_{\tau 2}\right|^{2}\right)\right] U_{\tau 1} U_{e 1}^{*}\right. \\
& -2 m_{1}^{2} \Delta_{32}\left|U_{\tau 1}\right|^{2} U_{\tau 3} U_{e 3}^{*}+2 m_{1} m_{2} \Delta_{31}\left(U_{e 1}^{*} U_{\tau 1}^{*} U_{\tau 2}^{2}+U_{e 2}^{*} U_{\tau 2}^{*} U_{\tau 1}^{2}\right) \\
& \left.+2 m_{1} m_{3} \Delta_{21}\left(U_{e 1}^{*} U_{\tau 1}^{*} U_{\tau 3}^{2}+U_{e 3}^{*} U_{\tau 3}^{*} U_{\tau 1}^{2}\right)\right\}, \\
U_{\tau 2}^{\prime} U_{e 2}^{*} \simeq & U_{\tau 2} U_{e 2}^{*}-\frac{\epsilon}{\Delta_{21} \Delta_{32}}\left\{\left[\left(m_{1}^{2} m_{3}^{2}-m_{2}^{4}\right)\left(1-2\left|U_{\tau 2}\right|^{2}\right)-m_{2}^{2} \Delta_{31}\left(1-2\left|U_{\tau 1}\right|^{2}\right)\right] U_{\tau 2} U_{e 2}^{*}\right. \\
& -2 m_{2}^{2} \Delta_{31}\left|U_{\tau 2}\right|^{2} U_{\tau 3} U_{e 3}^{*}+2 m_{1} m_{2} \Delta_{32}\left(U_{e 1}^{*} U_{\tau 1}^{*} U_{\tau 2}^{2}+U_{e 2}^{*} U_{\tau 2}^{*} U_{\tau 1}^{2}\right) \\
& \left.-2 m_{2} m_{3} \Delta_{21}\left(U_{e 2}^{*} U_{\tau 2}^{*} U_{\tau 3}^{2}+U_{e 3}^{*} U_{\tau 3}^{*} U_{\tau 2}^{2}\right)\right\}, \\
U_{\tau 3}^{\prime} U_{e 3}^{*} \simeq & U_{\tau 3} U_{e 3}^{*}+\frac{\epsilon}{\Delta_{31} \Delta_{32}}\left\{\left[\left(m_{1}^{2} m_{2}^{2}-m_{3}^{4}\right)\left(1-2\left|U_{\tau 3}\right|^{2}\right)+m_{3}^{2} \Delta_{21}\left(1-2\left|U_{\tau 2}\right|^{2}\right)\right] U_{\tau 3} U_{e 3}^{*}\right. \\
& +2 m_{3}^{2} \Delta_{21}\left|U_{\tau 3}\right|^{2} U_{\tau 1} U_{e 1}^{*}-2 m_{2} m_{3} \Delta_{31}\left(U_{e 2}^{*} U_{\tau 2}^{*} U_{\tau 3}^{2}+U_{e 3}^{*} U_{\tau 3}^{*} U_{\tau 2}^{2}\right) \\
& \left.-2 m_{1} m_{3} \Delta_{32}\left(U_{e 1}^{*} U_{\tau 1}^{*} U_{\tau 3}^{2}+U_{e 3}^{*} U_{\tau 3}^{*} U_{\tau 1}^{2}\right)\right\},
\end{aligned}
$$

for $\Delta_{\mu}^{\prime}$; and

$$
\begin{aligned}
U_{e 1}^{\prime} U_{\mu 1}^{\prime *} \simeq & U_{e 1} U_{\mu 1}^{*}-\frac{2 \epsilon}{\Delta_{21} \Delta_{31}}\left\{\left[\left(m_{2}^{2} m_{3}^{2}-m_{1}^{4}\right)\left|U_{\tau 1}\right|^{2}-m_{1}^{2} \Delta_{32}\left|U_{\tau 2}\right|^{2}\right] U_{e 1} U_{\mu 1}^{*}\right. \\
& -m_{1}^{2} \Delta_{32}\left(1-\left|U_{\tau 1}\right|^{2}\right) U_{e 3} U_{\mu 3}^{*}-m_{1} m_{2} \Delta_{31}\left(U_{e 1} U_{\tau 1} U_{\mu 2}^{*} U_{\tau 2}^{*}+U_{e 2} U_{\tau 2} U_{\mu 1}^{*} U_{\tau 1}^{*}\right) \\
& \left.-m_{1} m_{3} \Delta_{21}\left(U_{e 1} U_{\tau 1} U_{\mu 3}^{*} U_{\tau 3}^{*}+U_{e 3} U_{\tau 3} U_{\mu 1}^{*} U_{\tau 1}^{*}\right)\right\}, \\
U_{e 2}^{\prime} U_{\mu 2}^{\prime *} \simeq & U_{e 2} U_{\mu 2}^{*}+\frac{2 \epsilon}{\Delta_{21} \Delta_{32}}\left\{\left[\left(m_{1}^{2} m_{3}^{2}-m_{2}^{4}\right)\left|U_{\tau 2}\right|^{2}-m_{2}^{2} \Delta_{31}\left|U_{\tau 1}\right|^{2}\right] U_{e 2} U_{\mu 2}^{*}\right. \\
& -m_{2}^{2} \Delta_{31}\left(1-\left|U_{\tau 2}\right|^{2}\right) U_{e 3} U_{\mu 3}^{*}-m_{1} m_{2} \Delta_{32}\left(U_{e 1} U_{\tau 1} U_{\mu 2}^{*} U_{\tau 2}^{*}+U_{e 2} U_{\tau 2} U_{\mu 1}^{*} U_{\tau 1}^{*}\right) \\
& \left.+m_{2} m_{3} \Delta_{21}\left(U_{e 2} U_{\tau 2} U_{\mu 3}^{*} U_{\tau 3}^{*}+U_{e 3} U_{\tau 3} U_{\mu 2}^{*} U_{\tau 2}^{*}\right)\right\}, \\
U_{e 3}^{\prime} U_{\mu 3}^{\prime *} \simeq & U_{e 3} U_{\mu 3}^{*}-\frac{2 \epsilon}{\Delta_{31} \Delta_{32}}\left\{\left[\left(m_{1}^{2} m_{2}^{2}-m_{3}^{4}\right)\left|U_{\tau 3}\right|^{2}+m_{3}^{2} \Delta_{21}\left|U_{\tau 2}\right|^{2}\right] U_{e 3} U_{\mu 3}^{*}\right. \\
& +m_{3}^{2} \Delta_{21}\left(1-\left|U_{\tau 3}\right|^{2}\right) U_{e 1} U_{\mu 1}^{*}+m_{1} m_{3} \Delta_{32}\left(U_{e 1} U_{\tau 1} U_{\mu 3}^{*} U_{\tau 3}^{*}+U_{e 3} U_{\tau 3} U_{\mu 1}^{*} U_{\tau 1}^{*}\right) \\
& \left.+m_{2} m_{3} \Delta_{31}\left(U_{e 2} U_{\tau 2} U_{\mu 3}^{*} U_{\tau 3}^{*}+U_{e 3} U_{\tau 3} U_{\mu 2}^{*} U_{\tau 2}^{*}\right)\right\},
\end{aligned}
$$


for $\Delta_{\tau}^{\prime}$. The above analytical approximations of $\left|U_{\alpha i}^{\prime}\right|^{2}$ and $U_{\alpha i}^{\prime} U_{\beta i}^{\prime *}$ satisfy $\sum_{i}\left|U_{\alpha i}^{\prime}\right|^{2}=\sum_{\alpha}\left|U_{\alpha i}^{\prime}\right|^{2}=1$ and $\sum_{i} U_{\alpha i}^{\prime} U_{\beta i}^{\prime *}=0$. The LUTs $\Delta_{\alpha}^{\prime}$ of Majorana neutrinos can be fixed from Eqs. (34)-(36) though the vector sides $U_{\alpha i}^{\prime} U_{\alpha j}^{\prime *}$ of $\Delta_{i}^{\prime}$ cannot be derived in this way, implying that it is impossible to get any information on the Majorana phases at $\Lambda_{\mathrm{EW}}$. However, we can calculate $\left|U_{\alpha i}^{\prime} U_{\alpha j}^{\prime *}\right|^{2}$ from Eqs. (31)-(33) and fix the shapes of $\Delta_{i}^{\prime}$ without their orientations. With the help of Eq. (4) and the vector sides in Eqs. (34)-(36), the Jarlskog invariant $\mathcal{J}^{\prime}$ at $\Lambda_{\mathrm{EW}}$ for Majorana neutrinos can be given by

$$
\begin{aligned}
\mathcal{J}^{\prime} \simeq & \mathcal{J}-\frac{2 \epsilon}{\Delta_{21} \Delta_{31} \Delta_{32}}\left\{\mathcal { J } \left[m_{1}^{2}\left(m_{2}^{4}+m_{3}^{4}\right)\left(\left|U_{\tau 3}\right|^{2}-\left|U_{\tau 2}\right|^{2}\right)+m_{2}^{2}\left(m_{1}^{4}+m_{3}^{4}\right)\left(\left|U_{\tau 1}\right|^{2}-\left|U_{\tau 3}\right|^{2}\right)\right.\right. \\
& \left.+m_{3}^{2}\left(m_{1}^{4}+m_{2}^{4}\right)\left(\left|U_{\tau 2}\right|^{2}-\left|U_{\tau 1}\right|^{2}\right)\right]+m_{1} m_{2} \Delta_{31} \Delta_{32}\left[\left(\left|U_{\tau 2}\right|^{2}-\left|U_{\tau 1}\right|^{2}\right) \mathbb{I}_{e \tau}^{12}\right. \\
& \left.-\left(\left|U_{e 2}\right|^{2}-\left|U_{e 1}\right|^{2}\right) \mathbb{I}_{\tau \tau}^{12}\right]+m_{1} m_{3} \Delta_{21} \Delta_{32}\left[\left(\left|U_{\tau 1}\right|^{2}-\left|U_{\tau 3}\right|^{2}\right) \mathbb{I}_{e \tau}^{13}-\left(\left|U_{e 1}\right|^{2}-\left|U_{e 3}\right|^{2}\right) \mathbb{I}_{\tau \tau}^{13}\right] \\
& \left.+m_{2} m_{3} \Delta_{21} \Delta_{31}\left[\left(\left|U_{\tau 3}\right|^{2}-\left|U_{\tau 2}\right|^{2}\right) \mathbb{I}_{e \tau}^{23}-\left(\left|U_{e 3}\right|^{2}-\left|U_{e 2}\right|^{2}\right) \mathbb{I}_{\tau \tau}^{23}\right]\right\}
\end{aligned}
$$

where $\mathbb{I}_{\alpha \beta}^{i j}$ denote the imaginary parts of $U_{\alpha i} U_{\alpha j}^{*} U_{\beta i} U_{\beta j}^{*}$.

The nine inner angles of LUTs at $\Lambda_{\mathrm{EW}}$ running from $\Lambda_{\mathrm{H}}$ can be derived from Eqs. (34)-(36) and (21), and expressed as:

$$
\begin{aligned}
& \cot \phi_{e 1}^{\prime} \simeq \cot \phi_{e 1}+\frac{2 \eta_{\phi} \epsilon}{\mathcal{J} \Delta_{21} \Delta_{31} \Delta_{32}}\left\{\left|U_{\tau 2}\right|^{2}\left|U_{\tau 3}\right|^{2}\left(m_{2}^{2} \Delta_{31}^{2}\left|U_{\mu 3}\right|^{2}-m_{3}^{2} \Delta_{21}^{2}\left|U_{\mu 2}\right|^{2}\right)\right. \\
& +m_{1} m_{2} \Delta_{31} \Delta_{32}\left[\left(\left|U_{\tau 1}\right|^{2}+\left|U_{\tau 2}\right|^{2}\right) \mathbb{R}_{\mu \tau}^{12}+\left(\left|U_{\mu 1}\right|^{2}+\left|U_{\mu 2}\right|^{2}\right) \mathbb{R}_{\tau \tau}^{12}\right] \\
& +m_{1} m_{3} \Delta_{21} \Delta_{32}\left[\left(\left|U_{\tau 1}\right|^{2}+\left|U_{\tau 3}\right|^{2}\right) \mathbb{R}_{\mu \tau}^{13}+\left(\left|U_{\mu 1}\right|^{2}+\left|U_{\mu 3}\right|^{2}\right) \mathbb{R}_{\tau \tau}^{13}\right] \\
& +m_{2} m_{3} \Delta_{21} \Delta_{31}\left[\left(\left|U_{\tau 3}\right|^{2}-\left|U_{\tau 2}\right|^{2}\right) \mathbb{R}_{\mu \tau}^{23}+\left(\left|U_{\mu 3}\right|^{2}-\left|U_{\mu 2}\right|^{2}\right) \mathbb{R}_{\tau \tau}^{23}\right] \\
& +\cot \phi_{e 1}\left[m_{1} m_{2} \Delta_{31} \Delta_{32}\left(\left(\left|U_{\tau 1}\right|^{2}-\left|U_{\tau 2}\right|^{2}\right) \mathbb{I}_{\mu \tau}^{12}-\left(\left|U_{\mu 1}\right|^{2}-\left|U_{\mu 2}\right|^{2}\right) \mathbb{I}_{\tau \tau}^{12}\right)\right. \\
& +m_{1} m_{3} \Delta_{21} \Delta_{32}\left(\left(\left|U_{\tau 3}\right|^{2}-\left|U_{\tau 1}\right|^{2}\right) \mathbb{I}_{\mu \tau}^{13}-\left(\left|U_{\mu 3}\right|^{2}-\left|U_{\mu 1}\right|^{2}\right) \mathbb{I}_{\tau \tau}^{13}\right) \\
& \left.\left.+m_{2} m_{3} \Delta_{21} \Delta_{31}\left(\left(\left|U_{\tau 2}\right|^{2}-\left|U_{\tau 3}\right|^{2}\right) \mathbb{I}_{\mu \tau}^{23}-\left(\left|U_{\mu 2}\right|^{2}-\left|U_{\mu 3}\right|^{2}\right) \mathbb{I}_{\tau \tau}^{23}\right)\right]\right\}, \\
& \cot \phi_{e 2}^{\prime} \simeq \cot \phi_{e 2}+\frac{2 \eta_{\phi} \epsilon}{\mathcal{J} \Delta_{21} \Delta_{31} \Delta_{32}}\left\{\left|U_{\tau 1}\right|^{2}\left|U_{\tau 3}\right|^{2}\left(m_{3}^{2} \Delta_{21}^{2}\left|U_{\mu 1}\right|^{2}-m_{1}^{2} \Delta_{32}^{2}\left|U_{\mu 3}\right|^{2}\right)\right. \\
& -m_{1} m_{2} \Delta_{31} \Delta_{32}\left[\left(\left|U_{\tau 1}\right|^{2}+\left|U_{\tau 2}\right|^{2}\right) \mathbb{R}_{\mu \tau}^{12}+\left(\left|U_{\mu 1}\right|^{2}+\left|U_{\mu 2}\right|^{2}\right) \mathbb{R}_{\tau \tau}^{12}\right] \\
& +m_{1} m_{3} \Delta_{21} \Delta_{32}\left[\left(\left|U_{\tau 3}\right|^{2}-\left|U_{\tau 1}\right|^{2}\right) \mathbb{R}_{\mu \tau}^{13}+\left(\left|U_{\mu 3}\right|^{2}-\left|U_{\mu 1}\right|^{2}\right) \mathbb{R}_{\tau \tau}^{13}\right] \\
& +m_{2} m_{3} \Delta_{21} \Delta_{31}\left[\left(\left|U_{\tau 2}\right|^{2}+\left|U_{\tau 3}\right|^{2}\right) \mathbb{R}_{\mu \tau}^{23}+\left(\left|U_{\mu 2}\right|^{2}+\left|U_{\mu 3}\right|^{2}\right) \mathbb{R}_{\tau \tau}^{23}\right] \\
& +\cot \phi_{e 2}\left[m_{1} m_{2} \Delta_{31} \Delta_{32}\left(\left(\left|U_{\tau 1}\right|^{2}-\left|U_{\tau 2}\right|^{2}\right) \mathbb{I}_{\mu \tau}^{12}-\left(\left|U_{\mu 1}\right|^{2}-\left|U_{\mu 2}\right|^{2}\right) \mathbb{I}_{\tau \tau}^{12}\right)\right. \\
& +m_{1} m_{3} \Delta_{21} \Delta_{32}\left(\left(\left|U_{\tau 3}\right|^{2}-\left|U_{\tau 1}\right|^{2}\right) \mathbb{I}_{\mu \tau}^{13}-\left(\left|U_{\mu 3}\right|^{2}-\left|U_{\mu 1}\right|^{2}\right) \mathbb{I}_{\tau \tau}^{13}\right) \\
& \left.\left.+m_{2} m_{3} \Delta_{21} \Delta_{31}\left(\left(\left|U_{\tau 2}\right|^{2}-\left|U_{\tau 3}\right|^{2}\right) \mathbb{I}_{\mu \tau}^{23}-\left(\left|U_{\mu 2}\right|^{2}-\left|U_{\mu 3}\right|^{2}\right) \mathbb{I}_{\tau \tau}^{23}\right)\right]\right\}, \\
& \cot \phi_{e 3}^{\prime} \simeq \cot \phi_{e 3}+\frac{2 \eta_{\phi} \epsilon}{\mathcal{J} \Delta_{21} \Delta_{31} \Delta_{32}}\left\{\left|U_{\tau 1}\right|^{2}\left|U_{\tau 2}\right|^{2}\left(m_{1}^{2} \Delta_{32}^{2}\left|U_{\mu 2}\right|^{2}-m_{2}^{2} \Delta_{31}^{2}\left|U_{\mu 1}\right|^{2}\right)\right. \\
& +m_{1} m_{2} \Delta_{31} \Delta_{32}\left[\left(\left|U_{\tau 2}\right|^{2}-\left|U_{\tau 1}\right|^{2}\right) \mathbb{R}_{\mu \tau}^{12}+\left(\left|U_{\mu 2}\right|^{2}-\left|U_{\mu 1}\right|^{2}\right) \mathbb{R}_{\tau \tau}^{12}\right] \\
& -m_{1} m_{3} \Delta_{21} \Delta_{32}\left[\left(\left|U_{\tau 1}\right|^{2}+\left|U_{\tau 3}\right|^{2}\right) \mathbb{R}_{\mu \tau}^{13}+\left(\left|U_{\mu 1}\right|^{2}+\left|U_{\mu 3}\right|^{2}\right) \mathbb{R}_{\tau \tau}^{13}\right] \\
& -m_{2} m_{3} \Delta_{21} \Delta_{31}\left[\left(\left|U_{\tau 2}\right|^{2}+\left|U_{\tau 3}\right|^{2}\right) \mathbb{R}_{\mu \tau}^{23}+\left(\left|U_{\mu 2}\right|^{2}+\left|U_{\mu 3}\right|^{2}\right) \mathbb{R}_{\tau \tau}^{23}\right] \\
& +\cot \phi_{e 3}\left[m_{1} m_{2} \Delta_{31} \Delta_{32}\left(\left(\left|U_{\tau 1}\right|^{2}-\left|U_{\tau 2}\right|^{2}\right) \mathbb{I}_{\mu \tau}^{12}-\left(\left|U_{\mu 1}\right|^{2}-\left|U_{\mu 2}\right|^{2}\right) \mathbb{I}_{\tau \tau}^{12}\right)\right. \\
& +m_{1} m_{3} \Delta_{21} \Delta_{32}\left(\left(\left|U_{\tau 3}\right|^{2}-\left|U_{\tau 1}\right|^{2}\right) \mathbb{I}_{\mu \tau}^{13}-\left(\left|U_{\mu 3}\right|^{2}-\left|U_{\mu 1}\right|^{2}\right) \mathbb{I}_{\tau \tau}^{13}\right) \\
& \left.\left.+m_{2} m_{3} \Delta_{21} \Delta_{31}\left(\left(\left|U_{\tau 2}\right|^{2}-\left|U_{\tau 3}\right|^{2}\right) \mathbb{I}_{\mu \tau}^{23}-\left(\left|U_{\mu 2}\right|^{2}-\left|U_{\mu 3}\right|^{2}\right) \mathbb{I}_{\tau \tau}^{23}\right)\right]\right\} \text {; }
\end{aligned}
$$


and

$$
\begin{aligned}
& \cot \phi_{\mu 1}^{\prime} \simeq \cot \phi_{\mu 1}+\frac{2 \eta_{\phi} \epsilon}{\mathcal{J} \Delta_{21} \Delta_{31} \Delta_{32}}\left\{\left|U_{\tau 2}\right|^{2}\left|U_{\tau 3}\right|^{2}\left(m_{2}^{2} \Delta_{31}^{2}\left|U_{e 3}\right|^{2}-m_{3}^{2} \Delta_{21}^{2}\left|U_{e 2}\right|^{2}\right)\right. \\
& +m_{1} m_{2} \Delta_{31} \Delta_{32}\left[\left(\left|U_{\tau 1}\right|^{2}+\left|U_{\tau 2}\right|^{2}\right) \mathbb{R}_{e \tau}^{12}+\left(\left|U_{e 1}\right|^{2}+\left|U_{e 2}\right|^{2}\right) \mathbb{R}_{\tau \tau}^{12}\right] \\
& +m_{1} m_{3} \Delta_{21} \Delta_{32}\left[\left(\left|U_{\tau 1}\right|^{2}+\left|U_{\tau 3}\right|^{2}\right) \mathbb{R}_{e \tau}^{13}+\left(\left|U_{e 1}\right|^{2}+\left|U_{e 3}\right|^{2}\right) \mathbb{R}_{\tau \tau}^{13}\right] \\
& +m_{2} m_{3} \Delta_{21} \Delta_{31}\left[\left(\left|U_{\tau 3}\right|^{2}-\left|U_{\tau 2}\right|^{2}\right) \mathbb{R}_{e \tau}^{23}+\left(\left|U_{e 3}\right|^{2}-\left|U_{e 2}\right|^{2}\right) \mathbb{R}_{\tau \tau}^{23}\right] \\
& -\cot \phi_{\mu 1}\left[m_{1} m_{2} \Delta_{31} \Delta_{32}\left(\left(\left|U_{\tau 1}\right|^{2}-\left|U_{\tau 2}\right|^{2}\right) \mathbb{I}_{e \tau}^{12}-\left(\left|U_{e 1}\right|^{2}-\left|U_{e 2}\right|^{2}\right) \mathbb{I}_{\tau \tau}^{12}\right)\right. \\
& +m_{1} m_{3} \Delta_{21} \Delta_{32}\left(\left(\left|U_{\tau 3}\right|^{2}-\left|U_{\tau 1}\right|^{2}\right) \mathbb{I}_{e \tau}^{13}-\left(\left|U_{e 3}\right|^{2}-\left|U_{e 1}\right|^{2}\right) \mathbb{I}_{\tau \tau}^{13}\right) \\
& \left.\left.+m_{2} m_{3} \Delta_{21} \Delta_{31}\left(\left(\left|U_{\tau 2}\right|^{2}-\left|U_{\tau 3}\right|^{2}\right) \mathbb{I}_{e \tau}^{23}-\left(\left|U_{e 2}\right|^{2}-\left|U_{e 3}\right|^{2}\right) \mathbb{I}_{\tau \tau}^{23}\right)\right]\right\}, \\
& \cot \phi_{\mu 2}^{\prime} \simeq \cot \phi_{\mu 2}+\frac{2 \eta_{\phi} \epsilon}{\mathcal{J} \Delta_{21} \Delta_{31} \Delta_{32}}\left\{\left|U_{\tau 1}\right|^{2}\left|U_{\tau 3}\right|^{2}\left(m_{3}^{2} \Delta_{21}^{2}\left|U_{e 1}\right|^{2}-m_{1}^{2} \Delta_{32}^{2}\left|U_{e 3}\right|^{2}\right)\right. \\
& -m_{1} m_{2} \Delta_{31} \Delta_{32}\left[\left(\left|U_{\tau 1}\right|^{2}+\left|U_{\tau 2}\right|^{2}\right) \mathbb{R}_{e \tau}^{12}+\left(\left|U_{e 1}\right|^{2}+\left|U_{e 2}\right|^{2}\right) \mathbb{R}_{\tau \tau}^{12}\right] \\
& +m_{1} m_{3} \Delta_{21} \Delta_{32}\left[\left(\left|U_{\tau 3}\right|^{2}-\left|U_{\tau 1}\right|^{2}\right) \mathbb{R}_{e \tau}^{13}+\left(\left|U_{e 3}\right|^{2}-\left|U_{e 1}\right|^{2}\right) \mathbb{R}_{\tau \tau}^{13}\right] \\
& +m_{2} m_{3} \Delta_{21} \Delta_{31}\left[\left(\left|U_{\tau 2}\right|^{2}+\left|U_{\tau 3}\right|^{2}\right) \mathbb{R}_{e \tau}^{23}+\left(\left|U_{e 2}\right|^{2}+\left|U_{e 3}\right|^{2}\right) \mathbb{R}_{\tau \tau}^{23}\right] \\
& -\cot \phi_{\mu 2}\left[m_{1} m_{2} \Delta_{31} \Delta_{32}\left(\left(\left|U_{\tau 1}\right|^{2}-\left|U_{\tau 2}\right|^{2}\right) \mathbb{I}_{e \tau}^{12}-\left(\left|U_{e 1}\right|^{2}-\left|U_{e 2}\right|^{2}\right) \mathbb{I}_{\tau \tau}^{12}\right)\right. \\
& +m_{1} m_{3} \Delta_{21} \Delta_{32}\left(\left(\left|U_{\tau 3}\right|^{2}-\left|U_{\tau 1}\right|^{2}\right) \mathbb{I}_{e \tau}^{13}-\left(\left|U_{e 3}\right|^{2}-\left|U_{e 1}\right|^{2}\right) \mathbb{I}_{\tau \tau}^{13}\right) \\
& \left.\left.+m_{2} m_{3} \Delta_{21} \Delta_{31}\left(\left(\left|U_{\tau 2}\right|^{2}-\left|U_{\tau 3}\right|^{2}\right) \mathbb{I}_{e \tau}^{23}-\left(\left|U_{e 2}\right|^{2}-\left|U_{e 3}\right|^{2}\right) \mathbb{I}_{\tau \tau}^{23}\right)\right]\right\}, \\
& \cot \phi_{\mu 3}^{\prime} \simeq \cot \phi_{\mu 3}+\frac{2 \eta_{\phi} \epsilon}{\mathcal{J} \Delta_{21} \Delta_{31} \Delta_{32}}\left\{\left|U_{\tau 1}\right|^{2}\left|U_{\tau 2}\right|^{2}\left(m_{1}^{2} \Delta_{32}^{2}\left|U_{e 2}\right|^{2}-m_{2}^{2} \Delta_{31}^{2}\left|U_{e 1}\right|^{2}\right)\right. \\
& +m_{1} m_{2} \Delta_{31} \Delta_{32}\left[\left(\left|U_{\tau 2}\right|^{2}-\left|U_{\tau 1}\right|^{2}\right) \mathbb{R}_{e \tau}^{12}+\left(\left|U_{e 2}\right|^{2}-\left|U_{e 1}\right|^{2}\right) \mathbb{R}_{\tau \tau}^{12}\right] \\
& -m_{1} m_{3} \Delta_{21} \Delta_{32}\left[\left(\left|U_{\tau 1}\right|^{2}+\left|U_{\tau 3}\right|^{2}\right) \mathbb{R}_{e \tau}^{13}+\left(\left|U_{e 1}\right|^{2}+\left|U_{e 3}\right|^{2}\right) \mathbb{R}_{\tau \tau}^{13}\right] \\
& -m_{2} m_{3} \Delta_{21} \Delta_{31}\left[\left(\left|U_{\tau 2}\right|^{2}+\left|U_{\tau 3}\right|^{2}\right) \mathbb{R}_{e \tau}^{23}+\left(\left|U_{e 2}\right|^{2}+\left|U_{e 3}\right|^{2}\right) \mathbb{R}_{\tau \tau}^{23}\right] \\
& -\cot \phi_{\mu 3}\left[m_{1} m_{2} \Delta_{31} \Delta_{32}\left(\left(\left|U_{\tau 1}\right|^{2}-\left|U_{\tau 2}\right|^{2}\right) \mathbb{I}_{e \tau}^{12}-\left(\left|U_{e 1}\right|^{2}-\left|U_{e 2}\right|^{2}\right) \mathbb{I}_{\tau \tau}^{12}\right)\right. \\
& +m_{1} m_{3} \Delta_{21} \Delta_{32}\left(\left(\left|U_{\tau 3}\right|^{2}-\left|U_{\tau 1}\right|^{2}\right) \mathbb{I}_{e \tau}^{13}-\left(\left|U_{e 3}\right|^{2}-\left|U_{e 1}\right|^{2}\right) \mathbb{I}_{\tau \tau}^{13}\right) \\
& \left.\left.+m_{2} m_{3} \Delta_{21} \Delta_{31}\left(\left(\left|U_{\tau 2}\right|^{2}-\left|U_{\tau 3}\right|^{2}\right) \mathbb{I}_{e \tau}^{23}-\left(\left|U_{e 2}\right|^{2}-\left|U_{e 3}\right|^{2}\right) \mathbb{I}_{\tau \tau}^{23}\right)\right]\right\} \text {; }
\end{aligned}
$$

and

$$
\begin{aligned}
\cot \phi_{\tau 1}^{\prime} \simeq & \cot \phi_{\tau 1}+\frac{2 \eta_{\phi} \epsilon}{\mathcal{J} \Delta_{21} \Delta_{31} \Delta_{32}}\left\{m_{3}^{2} \Delta_{21}^{2}\left|U_{e 2}\right|^{2}\left|U_{\mu 2}\right|^{2}\left(1-\left|U_{\tau 3}\right|^{2}\right)-m_{2}^{2} \Delta_{31}^{2}\left|U_{e 3}\right|^{2}\left|U_{\mu 3}\right|^{2}\left(1-\left|U_{\tau 2}\right|^{2}\right)\right. \\
& +m_{1} m_{2} \Delta_{31} \Delta_{32}\left[\left(\left|U_{\mu 1}\right|^{2}+\left|U_{\mu 2}\right|^{2}\right) \mathbb{R}_{e \tau}^{12}+\left(\left|U_{e 1}\right|^{2}+\left|U_{e 2}\right|^{2}\right) \mathbb{R}_{\mu \tau}^{12}\right] \\
& +m_{1} m_{3} \Delta_{21} \Delta_{32}\left[\left(\left|U_{\mu 1}\right|^{2}+\left|U_{\mu 3}\right|^{2}\right) \mathbb{R}_{e \tau}^{13}+\left(\left|U_{e 1}\right|^{2}+\left|U_{e 3}\right|^{2}\right) \mathbb{R}_{\mu \tau}^{13}\right] \\
& +m_{2} m_{3} \Delta_{21} \Delta_{31}\left[\left(\left|U_{\mu 3}\right|^{2}-\left|U_{\mu 2}\right|^{2}\right) \mathbb{R}_{e \tau}^{23}+\left(\left|U_{e 3}\right|^{2}-\left|U_{e 2}\right|^{2}\right) \mathbb{R}_{\mu \tau}^{23}\right] \\
& +\cot \phi_{\tau 1}\left[m_{1} m_{2} \Delta_{31} \Delta_{32}\left(\left(\left|U_{\mu 1}\right|^{2}-\left|U_{\mu 2}\right|^{2}\right) \mathbb{I}_{e \tau}^{12}-\left(\left|U_{e 1}\right|^{2}-\left|U_{e 2}\right|^{2}\right) \mathbb{I}_{\mu \tau}^{12}\right)\right. \\
& +m_{1} m_{3} \Delta_{21} \Delta_{32}\left(\left(\left|U_{\mu 3}\right|^{2}-\left|U_{\mu 1}\right|^{2}\right) \mathbb{I}_{e \tau}^{13}-\left(\left|U_{e 3}\right|^{2}-\left|U_{e 1}\right|^{2}\right) \mathbb{I}_{\mu \tau}^{13}\right) \\
& \left.\left.+m_{2} m_{3} \Delta_{21} \Delta_{31}\left(\left(\left|U_{\mu 2}\right|^{2}-\left|U_{\mu 3}\right|^{2}\right) \mathbb{I}_{e \tau}^{23}-\left(\left|U_{e 2}\right|^{2}-\left|U_{e 3}\right|^{2}\right) \mathbb{I}_{\mu \tau}^{23}\right)\right]\right\}
\end{aligned}
$$




$$
\begin{aligned}
& \cot \phi_{\tau 2}^{\prime} \simeq \cot \phi_{\tau 2}+\frac{2 \eta_{\phi} \epsilon}{\mathcal{J} \Delta_{21} \Delta_{31} \Delta_{32}}\left\{m_{1}^{2} \Delta_{32}^{2}\left|U_{e 3}\right|^{2}\left|U_{\mu 3}\right|^{2}\left(1-\left|U_{\tau 1}\right|^{2}\right)-m_{3}^{2} \Delta_{21}^{2}\left|U_{e 1}\right|^{2}\left|U_{\mu 1}\right|^{2}\left(1-\left|U_{\tau 3}\right|^{2}\right)\right. \\
& -m_{1} m_{2} \Delta_{31} \Delta_{32}\left[\left(\left|U_{\mu 1}\right|^{2}+\left|U_{\mu 2}\right|^{2}\right) \mathbb{R}_{e \tau}^{12}+\left(\left|U_{e 1}\right|^{2}+\left|U_{e 2}\right|^{2}\right) \mathbb{R}_{\mu \tau}^{12}\right] \\
& +m_{1} m_{3} \Delta_{21} \Delta_{32}\left[\left(\left|U_{\mu 3}\right|^{2}-\left|U_{\mu 1}\right|^{2}\right) \mathbb{R}_{e \tau}^{13}+\left(\left|U_{e 3}\right|^{2}-\left|U_{e 1}\right|^{2}\right) \mathbb{R}_{\mu \tau}^{13}\right] \\
& +m_{2} m_{3} \Delta_{21} \Delta_{31}\left[\left(\left|U_{\mu 2}\right|^{2}+\left|U_{\mu 3}\right|^{2}\right) \mathbb{R}_{e \tau}^{23}+\left(\left|U_{e 2}\right|^{2}+\left|U_{e 3}\right|^{2}\right) \mathbb{R}_{\mu \tau}^{23}\right] \\
& +\cot \phi_{\tau 2}\left[m_{1} m_{2} \Delta_{31} \Delta_{32}\left(\left(\left|U_{\mu 1}\right|^{2}-\left|U_{\mu 2}\right|^{2}\right) \mathbb{I}_{e \tau}^{12}-\left(\left|U_{e 1}\right|^{2}-\left|U_{e 2}\right|^{2}\right) \mathbb{I}_{\mu \tau}^{12}\right)\right. \\
& +m_{1} m_{3} \Delta_{21} \Delta_{32}\left(\left(\left|U_{\mu 3}\right|^{2}-\left|U_{\mu 1}\right|^{2}\right) \mathbb{I}_{e \tau}^{13}-\left(\left|U_{e 3}\right|^{2}-\left|U_{e 1}\right|^{2}\right) \mathbb{I}_{\mu \tau}^{13}\right) \\
& \left.\left.+m_{2} m_{3} \Delta_{21} \Delta_{31}\left(\left(\left|U_{\mu 2}\right|^{2}-\left|U_{\mu 3}\right|^{2}\right) \mathbb{I}_{e \tau}^{23}-\left(\left|U_{e 2}\right|^{2}-\left|U_{e 3}\right|^{2}\right) \mathbb{I}_{\mu \tau}^{23}\right)\right]\right\}, \\
& \cot \phi_{\tau 3}^{\prime} \simeq \cot \phi_{\tau 3}+\frac{2 \eta_{\phi} \epsilon}{\mathcal{J} \Delta_{21} \Delta_{31} \Delta_{32}}\left\{m_{2}^{2} \Delta_{31}^{2}\left|U_{e 1}\right|^{2}\left|U_{\mu 1}\right|^{2}\left(1-\left|U_{\tau 2}\right|^{2}\right)-m_{1}^{2} \Delta_{32}^{2}\left|U_{e 2}\right|^{2}\left|U_{\mu 2}\right|^{2}\left(1-\left|U_{\tau 1}\right|^{2}\right)\right. \\
& +m_{1} m_{2} \Delta_{31} \Delta_{32}\left[\left(\left|U_{\mu 2}\right|^{2}-\left|U_{\mu 1}\right|^{2}\right) \mathbb{R}_{e \tau}^{12}+\left(\left|U_{e 2}\right|^{2}-\left|U_{e 1}\right|^{2}\right) \mathbb{R}_{\mu \tau}^{12}\right] \\
& -m_{1} m_{3} \Delta_{21} \Delta_{32}\left[\left(\left|U_{\mu 1}\right|^{2}+\left|U_{\mu 3}\right|^{2}\right) \mathbb{R}_{e \tau}^{13}+\left(\left|U_{e 1}\right|^{2}+\left|U_{e 3}\right|^{2}\right) \mathbb{R}_{\mu \tau}^{13}\right] \\
& -m_{2} m_{3} \Delta_{21} \Delta_{31}\left[\left(\left|U_{\mu 2}\right|^{2}+\left|U_{\mu 3}\right|^{2}\right) \mathbb{R}_{e \tau}^{23}+\left(\left|U_{e 2}\right|^{2}+\left|U_{e 3}\right|^{2}\right) \mathbb{R}_{\mu \tau}^{23}\right] \\
& +\cot \phi_{\tau 3}\left[m_{1} m_{2} \Delta_{31} \Delta_{32}\left(\left(\left|U_{\mu 1}\right|^{2}-\left|U_{\mu 2}\right|^{2}\right) \mathbb{I}_{e \tau}^{12}-\left(\left|U_{e 1}\right|^{2}-\left|U_{e 2}\right|^{2}\right) \mathbb{I}_{\mu \tau}^{12}\right)\right. \\
& +m_{1} m_{3} \Delta_{21} \Delta_{32}\left(\left(\left|U_{\mu 3}\right|^{2}-\left|U_{\mu 1}\right|^{2}\right) \mathbb{I}_{e \tau}^{13}-\left(\left|U_{e 3}\right|^{2}-\left|U_{e 1}\right|^{2}\right) \mathbb{I}_{\mu \tau}^{13}\right) \\
& \left.\left.+m_{2} m_{3} \Delta_{21} \Delta_{31}\left(\left(\left|U_{\mu 2}\right|^{2}-\left|U_{\mu 3}\right|^{2}\right) \mathbb{I}_{e \tau}^{23}-\left(\left|U_{e 2}\right|^{2}-\left|U_{e 3}\right|^{2}\right) \mathbb{I}_{\mu \tau}^{23}\right)\right]\right\} \text {. }
\end{aligned}
$$

Some discussions about the analytical results above for both Dirac and Majorana neutrinos are as follows:

(i) The approximate expressions of $\left|U_{e i}^{\prime}\right|^{2}$ and $U_{\mu i}^{\prime} U_{\tau i}^{\prime *}$ are similar to those of $\left|U_{\mu i}^{\prime}\right|^{2}$ and $U_{\tau i}^{\prime} U_{e i}^{\prime *}$, respectively. The analytical results for Majorana neutrinos are not equivalent to those for Dirac neutrinos even if one turns off the Majorana phases by setting their values to be zeros. In both cases, the corrections to the LUTs depend a lot on the magnitudes of the lightest neutrino mass and the small quantity $\epsilon$. The evolutions of the sides $U_{\alpha 3}^{\prime} U_{\beta 3}^{*}$ and the inner angles $\phi_{\alpha 3}$ are more stable against the RGE running.

(ii) Different from the Dirac case, $\mathcal{J}^{\prime}$ of Majorana neutrinos is in general nonzero even assuming $\mathcal{J}$ at $\Lambda_{\mathrm{H}}$ to be zero, and vice versa. One can conclude from Eq. (37) that there may exist leptonic $C P$ violation at $\Lambda_{\mathrm{EW}}$ unless all the Dirac and Majorana phases at the superhigh energy vanish. This observation is consistent with the analysis in Refs. [12,23].

(iii) The direct connections of the LUTs between two energy scales, which have been established above, are independent of the parametrization of $U$ and complementary to the differential forms in Ref. [12]. They can also reproduce the analytical approximations of neutrino masses, flavor mixing angles and the Dirac $C P$ phase in other references $[15,18,24]$ by taking a specific parametrization. Note that the accuracy of the approximate results above and in Sec. III will be very poor if the neutrino masses are strongly degenerate, i.e., the smallest neutrino mass is big enough. Considering the fact that the combination of Planck and baryon acoustic oscillation
(BAO) measurements gives the limit of the sum of three light neutrino masses as $\sum_{i} m_{i}<0.12 \mathrm{eV}$ at 95\% confidence level [25], one can use the analytical approximations to understand most part of the parameter space. We plan to explicitly study the case of nearly degenerate neutrino masses elsewhere.

\section{LUTS AND RGE-INDUCED $\mu$ - $\tau$ REFLECTION SYMMETRY BREAKING}

The $\mu-\tau$ reflection symmetry of the neutrino sector serving as the minimal discrete flavor symmetry to explain the lepton flavor mixing and $C P$ violation has been extensively studied for both Dirac and Majorana neutrinos [14]. One of the usual ways is that by assuming the $\mu-\tau$ reflection symmetry at a superhigh energy scale $\Lambda_{\mu \tau}$, we confront its RGE-induced breaking effects at $\Lambda_{\mathrm{EW}}$ with current experiment data $[14,18,24,26,27]$. This can be connected with the corresponding reformations of the LUTs below.

\section{A. The case of Dirac neutrinos}

If massive neutrinos are the Dirac particles, the $\mu-\tau$ reflection symmetry means that the effective Dirac neutrino mass term is invariant under the flavor and chargeconjugation transformations below:

$$
\begin{array}{cl}
\nu_{\mathrm{eL}} \leftrightarrow\left(\nu_{\mathrm{e}}\right)^{\mathrm{c}}, & \nu_{\mu \mathrm{L}} \leftrightarrow\left(\nu_{\tau \mathrm{L}}\right)^{\mathrm{c}}, \quad \nu_{\tau \mathrm{L}} \leftrightarrow\left(\nu_{\mu \mathrm{L}}\right)^{\mathrm{c}}, \\
N_{\mathrm{eR}} \leftrightarrow\left(N_{\mathrm{eR}}\right)^{\mathrm{c}}, & N_{\mu \mathrm{R}} \leftrightarrow\left(N_{\tau \mathrm{R}}\right)^{\mathrm{c}}, \quad N_{\tau \mathrm{R}} \leftrightarrow\left(N_{\mu \mathrm{R}}\right)^{\mathrm{c}},
\end{array}
$$

where $\nu_{\alpha \mathrm{L}}$ and $N_{\alpha \mathrm{R}}$ for $\alpha=e, \mu, \tau$ denote the left-handed and right-handed neutrino fields, respectively. This results 
in the constraint conditions of $\left(H_{\nu}\right)_{e \mu}=\left(H_{\nu}\right)_{e \tau}^{*}$ and $\left(H_{\nu}\right)_{\mu \mu}=$ $\left(H_{\nu}\right)_{\tau \tau}$ with $\left(H_{\nu}\right)_{\alpha \beta}=\sum_{i} m_{i}^{2} U_{\alpha i} U_{\beta i}^{*}$ being defined in subsection II A. To be specific, we have $U_{e i} U_{\mu i}^{*}=U_{e i}^{*} U_{\tau i}$ and $\left|U_{\mu i}\right|=\left|U_{\tau i}\right|$ for $i=1,2,3$, which can also be expressed as $U_{e i}=\eta_{i} U_{e i}^{*}$ and $U_{\mu i}=\eta_{i} U_{\tau i}^{*}$ with $\eta_{i}= \pm 1$. There are eight choices of $\left(\eta_{1}, \eta_{2}, \eta_{3}\right)$ while all of them are identical with one another through redefining the relevant phases of charged lepton and Dirac neutrino fields. Given the $\mu-\tau$ reflection symmetry of Dirac neutrinos at a superhigh energy scale $\Lambda_{\mu \tau}$, we have $\left|U_{\mu i}\right|=\left|U_{\tau i}\right|$. Hence the corresponding $\Delta_{i}$ are isosceles triangles, each with two equal sides $\left|U_{\mu j} U_{\mu k}^{*}\right|=\left|U_{\tau j} U_{\tau k}^{*}\right|$; and the two LUTs $\Delta_{\mu}$ and $\Delta_{\tau}$ are congruent with each other with three pairs of equal sides $\left|U_{i i} U_{e i}^{*}\right|=\left|U_{e i} U_{\mu i}^{*}\right|$. The deviations of the LUTs at $\Lambda_{\mathrm{EW}}$ from these special shapes at $\Lambda_{\mu \tau}$ due to the RGE running can demonstrate the RGE-induced $\mu$ - $\tau$ reflection symmetry breaking intuitively. Let us define

$$
\begin{aligned}
& \mathcal{S}_{\Delta_{1}}^{\mu \tau} \equiv\left|U_{\mu 2}^{\prime} U_{\mu 3}^{\prime *}\right|^{2}-\left|U_{\tau 2}^{\prime} U_{\tau 3}^{\prime *}\right|^{2}, \\
& \mathcal{S}_{\Delta_{2}}^{\mu \tau} \equiv\left|U_{\mu 3}^{\prime} U_{\mu 1}^{\prime *}\right|^{2}-\left|U_{\tau 3}^{\prime} U_{\tau 1}^{\prime *}\right|^{2}, \\
& \mathcal{S}_{\Delta_{3}}^{\mu \tau} \equiv\left|U_{\mu 1}^{\prime} U_{\mu 2}^{\prime *}\right|^{2}-\left|U_{\tau 1}^{\prime} U_{\tau 2}^{\prime *}\right|^{2},
\end{aligned}
$$

to describe the deviations of $\Delta_{i}^{\prime}$ from their $\mu-\tau$ reflection symmetry limits, and

$$
\begin{aligned}
& \mathcal{S}_{\Delta_{\mu \tau}}^{1} \equiv\left|U_{\tau 1}^{\prime} U_{e 1}^{\prime *}\right|^{2}-\left|U_{e 1}^{\prime} U_{\mu 1}^{\prime *}\right|^{2}, \\
& \mathcal{S}_{\Delta_{\mu \tau}}^{2} \equiv\left|U_{\tau 2}^{\prime} U_{e 2}^{*}\right|^{2}-\left|U_{e 2}^{\prime} U_{\mu 2}^{\prime *}\right|^{2}, \\
& \mathcal{S}_{\Delta_{\mu \tau}}^{3} \equiv\left|U_{\tau 3}^{\prime} U_{e 3}^{\prime *}\right|^{2}-\left|U_{e 3}^{\prime} U_{\mu 3}^{\prime *}\right|^{2},
\end{aligned}
$$

to show how the LUTs $\Delta_{\mu}^{\prime}$ and $\Delta_{\tau}^{\prime}$ can be reformed as compared with their $\mu-\tau$ reflection symmetry limits. With the help of $\left|U_{\mu i}\right|=\left|U_{\tau i}\right|$ together with Eqs. (14)-(16), the analytical approximations of the six asymmetries in Eqs. (42) and (43) can be expressed as:

$$
\begin{aligned}
\mathcal{S}_{\Delta_{1}}^{\mu \tau} \simeq & \frac{\epsilon}{\Delta_{21} \Delta_{31} \Delta_{32}}\left[m_{2}^{2} \Delta_{31}^{2}\left|U_{e 3}\right|^{2}\left(1-\left|U_{e 3}\right|^{2}\right)\right. \\
& \left.-m_{3}^{2} \Delta_{21}^{2}\left|U_{e 2}\right|^{2}\left(1-\left|U_{e 2}\right|^{2}\right)\right], \\
\mathcal{S}_{\Delta_{2}}^{\mu \tau} \simeq & \frac{\epsilon}{\Delta_{21} \Delta_{31} \Delta_{32}}\left[m_{3}^{2} \Delta_{21}^{2}\left|U_{e 1}\right|^{2}\left(1-\left|U_{e 1}\right|^{2}\right)\right. \\
& \left.-m_{1}^{2} \Delta_{32}^{2}\left|U_{e 3}\right|^{2}\left(1-\left|U_{e 3}\right|^{2}\right)\right], \\
\mathcal{S}_{\Delta_{3}}^{\mu \tau} \simeq & \frac{\epsilon}{\Delta_{21} \Delta_{31} \Delta_{32}}\left[m_{1}^{2} \Delta_{32}^{2}\left|U_{e 2}\right|^{2}\left(1-\left|U_{e 2}\right|^{2}\right)\right. \\
& \left.-m_{2}^{2} \Delta_{31}^{2}\left|U_{e 1}\right|^{2}\left(1-\left|U_{e 1}\right|^{2}\right)\right] ;
\end{aligned}
$$

and

$$
\begin{aligned}
\mathcal{S}_{\Delta_{\mu \tau}}^{1} \simeq & \frac{\epsilon\left|U_{e 1}\right|^{2}}{\Delta_{21} \Delta_{31}}\left[\left(m_{2}^{2} m_{3}^{2}-m_{1}^{4}\right)\left(1-\left|U_{e 1}\right|^{2}\right)\right. \\
& \left.-m_{1}^{2} \Delta_{32}\left(\left|U_{e 2}\right|^{2}-\left|U_{e 3}\right|^{2}\right)\right], \\
\mathcal{S}_{\Delta_{\mu \tau}}^{2} \simeq & -\frac{\epsilon\left|U_{e 2}\right|^{2}}{\Delta_{21} \Delta_{32}}\left[\left(m_{1}^{2} m_{3}^{2}-m_{2}^{4}\right)\left(1-\left|U_{e 2}\right|^{2}\right)\right. \\
& \left.-m_{2}^{2} \Delta_{31}\left(\left|U_{e 1}\right|^{2}-\left|U_{e 3}\right|^{2}\right)\right], \\
\mathcal{S}_{\Delta_{\mu \tau}}^{3} \simeq & \frac{\epsilon\left|U_{e 3}\right|^{2}}{\Delta_{31} \Delta_{32}}\left[\left(m_{1}^{2} m_{2}^{2}-m_{3}^{4}\right)\left(1-\left|U_{e 3}\right|^{2}\right)\right. \\
& \left.-m_{3}^{2} \Delta_{21}\left(\left|U_{e 1}\right|^{2}-\left|U_{e 2}\right|^{2}\right)\right],
\end{aligned}
$$

where $\left|U_{\mu i}\right|^{2}=\left|U_{\tau i}\right|^{2}$ has been be replaced by $\left(1-\left|U_{e i}\right|^{2}\right) / 2$. We can see that $\mathcal{S}_{\Delta_{\mu \tau}}^{1}$ and $\mathcal{S}_{\Delta_{\mu \tau}}^{2}$ are most sensitive to the neutrino mass ordering. The absolute values of $\mathcal{S}_{\Delta_{3}}^{\mu \tau}$ and $\mathcal{S}_{\Delta_{\mu \tau}}^{3}$ should be smaller because of the smallness of $\Delta_{21}$ and $\left|U_{e 3}\right|^{2}$. The Jarlskog invariant $\mathcal{J}^{\prime}$ at $\Lambda_{\mathrm{EW}}$ running from $\Lambda_{\mu \tau}$ can be written as

$$
\begin{aligned}
\mathcal{J}^{\prime} \simeq & \mathcal{J}-\frac{\epsilon \mathcal{J}}{\Delta_{21} \Delta_{31} \Delta_{32}}\left[m_{1}^{2}\left(m_{2}^{4}+m_{3}^{4}\right)\left(\left|U_{e 2}\right|^{2}-\left|U_{e 3}\right|^{2}\right)\right. \\
& -m_{2}^{2}\left(m_{1}^{4}+m_{3}^{4}\right)\left(\left|U_{e 1}\right|^{2}-\left|U_{e 3}\right|^{2}\right) \\
& \left.+m_{3}^{2}\left(m_{1}^{4}+m_{2}^{4}\right)\left(\left|U_{e 1}\right|^{2}-\left|U_{e 2}\right|^{2}\right)\right],
\end{aligned}
$$

whose magnitude is proportional to the area of the LUTs at $\Lambda_{\mathrm{EW}}$. Taking account of

$$
\cot \phi_{\alpha i}^{\prime}-\cot \phi_{\beta i}^{\prime}=\frac{\eta_{\phi}}{\mathcal{J}^{\prime}}\left(\left|U_{\alpha j}^{\prime} U_{\alpha k}^{\prime *}\right|^{2}-\left|U_{\beta j}^{\prime} U_{\beta k}^{\prime *}\right|^{2}\right),
$$

one obtains $\left(\cot \phi_{\tau i}^{\prime}-\cot \phi_{\mu i}^{\prime}\right) \simeq-\eta_{\phi} S_{\Delta_{i}}^{\mu \tau} / \mathcal{J}$, where only the first order of $\epsilon$ is kept and $S_{\Delta_{i}}^{\mu \tau}$ have been shown in Eq. (44). Noticing that bigger $\phi_{\mu i}^{\prime}-\phi_{\tau i}^{\prime}$ lead to bigger $\left(\cot \phi_{\tau i}^{\prime}-\cot \phi_{\mu i}^{\prime}\right)$ and $S_{\Delta_{i}}^{\mu \tau}$, we can also use the more intuitive asymmetries $\phi_{\mu i}^{\prime}-\phi_{\tau i}^{\prime}$ to replace $S_{\Delta_{i}}^{\mu \tau}$. The asymmetries of these three pairs of inner angles satisfy $\sum_{i}\left(\phi_{\mu i}^{\prime}-\phi_{\tau i}^{\prime}\right)=0$.

\section{B. The case of Majorana neutrinos}

When it comes to the Majorana neutrinos, the $\mu-\tau$ reflection symmetry implies the effective Majorana mass term should stay unchanged under the flavor and chargeconjugation transformations of neutrino fields: $\nu_{\mathrm{eL}} \leftrightarrow \nu_{\mathrm{eR}}^{\mathrm{c}}$, $\nu_{\mu \mathrm{L}} \leftrightarrow \nu_{\tau \mathrm{R}}^{\mathrm{c}}$, and $\nu_{\tau \mathrm{L}} \leftrightarrow \nu_{\mu \mathrm{R}}^{\mathrm{c}}$. This results in the limits to the elements of neutrino mass matrix $M_{\nu}:\left(M_{\nu}\right)_{e e}=\left(M_{\nu}\right)_{e e}^{*}$, $\left(M_{\nu}\right)_{e \mu}=\left(M_{\nu}\right)_{e \tau}^{*},\left(M_{\nu}\right)_{\mu \mu}=\left(M_{\tau}\right)_{\tau \tau}^{*}$, and $\left(M_{\nu}\right)_{\mu \tau}=\left(M_{\nu}\right)_{\mu \tau}^{*}$ with $\left(M_{\nu}\right)_{\alpha \beta} \equiv m_{i} U_{\alpha i} U_{\beta i}$ being defined in subsection II B. Furthermore, the constraint conditions can be expressed as $U_{e i}=\eta_{i} U_{e i}^{*}$ and $U_{\mu i}=\eta_{i} U_{\tau i}^{*}$ with $\eta_{i}= \pm 1$. Four of the eight choices of $\left(\eta_{1}, \eta_{2}, \eta_{3}\right)$ are independent because we can not redefine the Majorana neutrino fields to change the sign of arbitrary column of $U$ just like the Dirac case. Given the 
$\mu$ - $\tau$ reflection symmetry at $\Lambda_{\mu \tau}$, one gets $\left|U_{\mu i}\right|=\left|U_{\tau i}\right|$, which results in three isosceles LUTs $\Delta_{i}$ with $\left|U_{\mu j} U_{\mu k}^{*}\right|=$ $\left|U_{\tau j} U_{\tau k}^{*}\right|$ and a pair of congruent triangles $\left(\Delta_{\mu}\right.$ and $\left.\Delta_{\tau}\right)$ with $\left|U_{\tau i} U_{e i}^{*}\right|=\left|U_{e i} U_{\mu i}^{*}\right|$ just as the Dirac case. So the asymmetries defined in Eqs. (42) and (43) can be used to denote the deviations of LUTs of the Majorana neutrinos at $\Lambda_{\mathrm{EW}}$ from their special shapes at $\Lambda_{\mu \tau}$. The analytical approximations of these asymmetries in this case can be obtained with the help of $U_{\mu i}=\eta_{i} U_{\tau i}^{*}$ and Eqs. (31)-(33). The results are

$$
\begin{aligned}
\mathcal{S}_{\Delta_{1}}^{\mu \tau} \simeq & \frac{\epsilon}{\Delta_{21} \Delta_{31} \Delta_{32}}\left[\eta_{2} m_{2} \Delta_{31}\left(\eta_{2} m_{2} \Delta_{31}-\eta_{1} m_{1} \Delta_{32}\right)\left|U_{e 3}\right|^{2}\left(1-\left|U_{e 3}\right|^{2}\right)-\eta_{3} m_{3} \Delta_{21}\left(\eta_{1} m_{1} \Delta_{32}\right.\right. \\
& \left.\left.+\eta_{3} m_{3} \Delta_{21}\right)\left|U_{e 2}\right|^{2}\left(1-\left|U_{e 2}\right|^{2}\right)+\eta_{2} \eta_{3} m_{2} m_{3} \Delta_{21} \Delta_{31}\left(\left|U_{e 2}\right|^{2}-\left|U_{e 3}\right|^{2}\right)\left|U_{e 1}\right|^{2}\right], \\
\mathcal{S}_{\Delta_{2}}^{\mu \tau} \simeq & \frac{\epsilon}{\Delta_{21} \Delta_{31} \Delta_{32}}\left[\eta_{3} m_{3} \Delta_{21}\left(\eta_{3} m_{3} \Delta_{21}-\eta_{2} m_{2} \Delta_{31}\right)\left|U_{e 1}\right|^{2}\left(1-\left|U_{e 1}\right|^{2}\right)+\eta_{1} m_{1} \Delta_{32}\left(\eta_{2} m_{2} \Delta_{31}\right.\right. \\
& \left.\left.-\eta_{1} m_{1} \Delta_{32}\right)\left|U_{e 3}\right|^{2}\left(1-\left|U_{e 3}\right|^{2}\right)+\eta_{1} \eta_{3} m_{1} m_{3} \Delta_{21} \Delta_{32}\left(\left|U_{e 1}\right|^{2}-\left|U_{e 3}\right|^{2}\right)\left|U_{e 2}\right|^{2}\right], \\
\mathcal{S}_{\Delta_{3}}^{\mu \tau} \simeq & \frac{\epsilon}{\Delta_{21} \Delta_{31} \Delta_{32}}\left[\eta_{1} m_{1} \Delta_{32}\left(\eta_{1} m_{1} \Delta_{32}+\eta_{3} m_{3} \Delta_{21}\right)\left|U_{e 2}\right|^{2}\left(1-\left|U_{e 2}\right|^{2}\right)+\eta_{2} m_{2} \Delta_{31}\left(\eta_{3} m_{3} \Delta_{21}\right.\right. \\
& \left.\left.-\eta_{2} m_{2} \Delta_{31}\right)\left|U_{e 1}\right|^{2}\left(1-\left|U_{e 1}\right|^{2}\right)+\eta_{1} \eta_{2} m_{1} m_{2} \Delta_{31} \Delta_{32}\left(\left|U_{e 1}\right|^{2}-\left|U_{e 2}\right|^{2}\right)\left|U_{e 3}\right|^{2}\right],
\end{aligned}
$$

demonstrating the deviations of $\Delta_{i}^{\prime}$ at $\Lambda_{\mathrm{EW}}$ from their isosceles shapes at $\Lambda_{\mu \tau}$; and

$$
\begin{aligned}
& \mathcal{S}_{\Delta_{\mu \tau}}^{1} \simeq \frac{\epsilon\left|U_{e 1}\right|^{2}}{\Delta_{21} \Delta_{31}}\left[\left(m_{2}^{2} m_{3}^{2}-m_{1}^{4}\right)\left(1-\left|U_{e 1}\right|^{2}\right)-m_{1}^{2} \Delta_{32}\left(\left|U_{e 2}\right|^{2}-\left|U_{e 3}\right|^{2}\right)-2 \eta_{1} m_{1}\left(\eta_{2} m_{2} \Delta_{31}\left|U_{e 3}\right|^{2}+\eta_{3} m_{3} \Delta_{21}\left|U_{e 2}\right|^{2}\right)\right], \\
& \mathcal{S}_{\Delta_{\mu \tau}}^{2} \simeq-\frac{\epsilon\left|U_{e 2}\right|^{2}}{\Delta_{21} \Delta_{32}}\left[\left(m_{1}^{2} m_{3}^{2}-m_{2}^{4}\right)\left(1-\left|U_{e 2}\right|^{2}\right)-m_{2}^{2} \Delta_{31}\left(\left|U_{e 1}\right|^{2}-\left|U_{e 3}\right|^{2}\right)-2 \eta_{2} m_{2}\left(\eta_{1} m_{1} \Delta_{32}\left|U_{e 3}\right|^{2}-\eta_{3} m_{3} \Delta_{21}\left|U_{e 1}\right|^{2}\right)\right], \\
& \mathcal{S}_{\Delta_{\mu \tau}}^{3} \simeq \frac{\epsilon\left|U_{e 3}\right|^{2}}{\Delta_{31} \Delta_{32}}\left[\left(m_{1}^{2} m_{2}^{2}-m_{3}^{4}\right)\left(1-\left|U_{e 3}\right|^{2}\right)-m_{3}^{2} \Delta_{21}\left(\left|U_{e 1}\right|^{2}-\left|U_{e 2}\right|^{2}\right)+2 \eta_{3} m_{3}\left(\eta_{1} m_{1} \Delta_{32}\left|U_{e 2}\right|^{2}+\eta_{2} m_{2} \Delta_{31}\left|U_{e 1}\right|^{2}\right)\right],
\end{aligned}
$$

showing the deviations of $\Delta_{\mu}^{\prime}$ and $\Delta_{\tau}^{\prime}$ at $\Lambda_{\mathrm{EW}}$ from their congruent shapes at $\Lambda_{\mu \tau}$. From Eqs. (48) and (49), we find that $\mathcal{S}_{\Delta_{\mu \tau}}^{1}$ and $\mathcal{S}_{\Delta_{\mu \tau}}^{2}$ are most sensitive to the neutrino mass ordering; $\mathcal{S}_{\Delta_{\mu \tau}}^{3}$ and $\mathcal{S}_{\Delta_{3}}^{\mu \tau}$ are smaller due to the suppression of $\Delta_{21}$ and $\left|U_{e 3}\right|^{2}$. This conclusion is the same as the Dirac case. The connection of the Jarlskog invariants of Majorana neutrinos between $\Lambda_{\mathrm{EW}}$ and $\Lambda_{\mu \tau}$ can be written as

$$
\begin{aligned}
\mathcal{J}^{\prime} \simeq & \mathcal{J}-\frac{\epsilon \mathcal{J}}{\Delta_{21} \Delta_{31} \Delta_{32}}\left\{\left[m_{1}^{2}\left(m_{2}^{4}+m_{3}^{4}\right)-\eta_{2} \eta_{3} m_{2} m_{3} \Delta_{21} \Delta_{31}\right]\left(\left|U_{e 2}\right|^{2}-\left|U_{e 3}\right|^{2}\right)\right. \\
& -\left[m_{2}^{2}\left(m_{1}^{4}+m_{3}^{4}\right)+\eta_{1} \eta_{3} m_{1} m_{3} \Delta_{21} \Delta_{32}\right]\left(\left|U_{e 1}\right|^{2}-\left|U_{e 3}\right|^{2}\right) \\
& \left.+\left[m_{3}^{2}\left(m_{1}^{4}+m_{2}^{4}\right)-\eta_{1} \eta_{2} m_{1} m_{2} \Delta_{31} \Delta_{32}\right]\left(\left|U_{e 1}\right|^{2}-\left|U_{e 2}\right|^{2}\right)\right\}
\end{aligned}
$$

From Eqs. (47), (48), and (50), we can get $\left(\cot \phi_{\tau i}^{\prime}-\cot \phi_{\mu i}^{\prime}\right) \simeq-\eta_{\phi} S_{\Delta_{i}}^{\mu \tau} / \mathcal{J}$. The magnitude of $\left(\cot \phi_{\tau i}^{\prime}-\cot \phi_{\mu i}^{\prime}\right)$ always keeps consistent with that of $\phi_{\mu i}^{\prime}-\phi_{\tau i}^{\prime}$ or $S_{\Delta_{i}}^{\mu \tau}$.

It is clear to see that the analytical approximations of $\mathcal{S}_{\Delta_{i}}^{\mu \tau}, \mathcal{S}_{\Delta_{\mu \tau}}^{i}$, and $\mathcal{J}^{\prime}$ for the Majorana neutrinos include more odd terms of $\eta_{i}$ (i.e., $\eta_{i} \eta_{j}$ for $i \neq j$ ) compared with their counterparts for the Dirac neutrinos. These terms can be directly connected with the Majorana phases and have complicated influence on the LUT reformations at $\Lambda_{\mathrm{EW}}$.

\section{NUMERICAL ANALYSIS}

Before we start the numerical analysis, let us first parametrize $U$ as

$$
U=\left(\begin{array}{ccc}
c_{12} c_{13} & s_{12} c_{13} & s_{13} e^{-\mathrm{i} \delta} \\
-s_{12} c_{23}-c_{12} s_{13} s_{23} e^{\mathrm{i} \delta} & c_{12} c_{23}-s_{12} s_{13} s_{23} e^{\mathrm{i} \delta} & c_{13} s_{23} \\
-s_{12} s_{23}+c_{12} s_{13} c_{23} e^{\mathrm{i} \delta} & c_{12} s_{23}+s_{12} s_{13} c_{23} e^{\mathrm{i} \delta} & -c_{13} c_{23}
\end{array}\right)
$$


TABLE I. The correspondences between $(\delta, \rho, \sigma)$ and $\left(\eta_{1}, \eta_{2}, \eta_{3}\right)$ in the $\mu-\tau$ reflection symmetry limit for the Majorana neutrinos.

\begin{tabular}{cc}
\hline \hline$(\delta, \rho, \sigma)$ & $\left(\eta_{1}, \eta_{2}, \eta_{3}\right)$ \\
\hline$\left( \pm \frac{\pi}{2}, 0,0\right)$ & $(1,1,-1)$ \\
$\left( \pm \frac{\pi}{2}, \frac{\pi}{2}, 0\right)$ & $(-1,1,-1)$ \\
$\left( \pm \frac{\pi}{2}, 0, \frac{\pi}{2}\right)$ & $(1,-1,-1)$ \\
$\left( \pm \frac{\pi}{2}, \frac{\pi}{2}, \frac{\pi}{2}\right)$ & $(-1,-1,-1)$ \\
\hline
\end{tabular}

for the Dirac neutrinos with $c_{i j} \equiv \cos \theta_{i j}$ and $s_{i j} \equiv \sin \theta_{i j}$. For the Majorana neutrinos, one has to add the Majorana phase matrix $P_{\nu} \equiv \operatorname{Diag}\left\{e^{i \rho}, e^{i \sigma}, 1\right\}$ on the right side of Eq. (51). $U^{\prime}$ at $\Lambda_{\mathrm{EW}}$ has the same form as $U$ with the corresponding set of flavor mixing angles and $C P$ phases $\left(\theta_{12}^{\prime}, \theta_{13}^{\prime}, \theta_{23}^{\prime}, \delta^{\prime}, \rho^{\prime}, \sigma^{\prime}\right)$. According to the specific parametrization of $U$ in Eq. (51), we interpret the constraints of the $\mu-\tau$ reflection symmetry as two conditions for the Dirac neutrinos: $\theta_{23}=\pi / 4$ and $\delta= \pm \pi / 2$, and four conditions for the Majorana neutrinos: $\theta_{23}=\pi / 4, \delta= \pm \pi / 2, \rho=0$,

TABLE II. The numerical analysis of deviations of the six LUTs at $\Lambda_{\mathrm{EW}}$ from their $\mu$ - $\tau$ reflection symmetry limits at $\Lambda_{\mu \tau}$ for the Dirac neutrinos in the framework of the MSSM, by inputting $\left(\theta_{23}, \delta\right)=(\pi / 4,-\pi / 2)$ at $\Lambda_{\mu \tau}$ and allowing the smallest neutrino mass ( $m_{1}^{\prime}$ for the NMO case and $m_{3}^{\prime}$ for the IMO case) and the MSSM parameter $\tan \beta$ to vary in the ranges $[0,0.1] \mathrm{eV}$ and $[10,50]$, respectively.

\begin{tabular}{|c|c|c|c|c|}
\hline & \multicolumn{2}{|c|}{ Normal mass ordering (NMO) } & \multicolumn{2}{|c|}{ Inverted mass ordering (IMO) } \\
\hline & $\begin{array}{c}\text { Best-fit } \\
\chi_{\min }^{2} \simeq 0.01\end{array}$ & $\begin{array}{c}3 \sigma \text { range } \\
\chi^{2} \leq 9\end{array}$ & $\begin{array}{c}\text { Best-fit } \\
\chi_{\min }^{2} \simeq 7.94\end{array}$ & $\begin{array}{c}3 \sigma \text { range } \\
\chi^{2} \leq 9\end{array}$ \\
\hline$S_{\Delta_{\mu \tau}}^{1} / 10^{-2}$ & 6.60 & $(0.03,13.73)$ & 0.11 & $(0.10,0.64)$ \\
\hline$S_{\Delta_{\mu \tau}}^{2} / 10^{-2}$ & 0.25 & $(-0.15,1.34)$ & -0.08 & $(-0.52,-0.08)$ \\
\hline$S_{\Delta_{\mu \tau}}^{3} / 10^{-3}$ & -2.26 & $(-5.16,-0.03)$ & 0.03 & $(0.03,0.18)$ \\
\hline$\phi_{\mu 1}^{\prime}-\phi_{\tau 1}^{\prime}$ & $59.45^{\circ}$ & $\left(0.03^{\circ}, 178.71^{\circ}\right)$ & $1.60^{\circ}$ & $\left(1.55^{\circ}, 9.79^{\circ}\right)$ \\
\hline$\phi_{\mu 2}^{\prime}-\phi_{\tau 2}^{\prime}$ & $-54.09^{\circ}$ & $\left(-178.53^{\circ}, 0.60^{\circ}\right)$ & $-1.58^{\circ}$ & $\left(-9.70^{\circ},-1.54^{\circ}\right)$ \\
\hline$\phi_{\mu 3}^{\prime}-\phi_{\tau 3}^{\prime}$ & $-5.36^{\circ}$ & $\left(-8.20^{\circ},-0.05^{\circ}\right)$ & $-0.02^{\circ}$ & $\left(-0.10^{\circ}, 0.07^{\circ}\right)$ \\
\hline $\mathcal{J}^{\prime} / 10^{-2}$ & -2.85 & $(-3.48,-0.04)$ & -3.32 & $(-3.39,-3.25)$ \\
\hline$m_{1}^{\prime}$ or $m_{3}^{\prime} / \mathrm{eV}$ & 0.085 & $(0,0.1)$ & 0.001 & $(0,0.078)$ \\
\hline $\tan \beta$ & 32 & $(10,50)$ & 10 & $(10,24)$ \\
\hline
\end{tabular}

TABLE III. The numerical analysis of deviations of the six LUTs at $\Lambda_{\mathrm{EW}}$ from their $\mu-\tau$ reflection symmetry limits at $\Lambda_{\mu \tau}$ for the Majorana neutrinos in the framework of the MSSM, by inputting $\left(\theta_{23}, \delta, \rho, \sigma\right)=(\pi / 4,-\pi / 2,0,0)$ at $\Lambda_{\mu \tau}$ and allowing the smallest neutrino mass at $\Lambda_{\mathrm{EW}}\left(m_{1}^{\prime}\right.$ for the NMO case and $m_{3}^{\prime}$ for the IMO case) and the MSSM parameter $\tan \beta$ to vary in the ranges $[0,0.1] \mathrm{eV}$ and $[10,50]$, respectively.

\begin{tabular}{|c|c|c|c|c|}
\hline & \multicolumn{2}{|c|}{ Normal mass ordering (NMO) } & \multicolumn{2}{|c|}{ Inverted mass ordering (IMO) } \\
\hline & $\begin{array}{c}\text { Best-fit } \\
\chi_{\min }^{2} \simeq 0.77 \\
\end{array}$ & $\begin{array}{c}3 \sigma \text { range } \\
\chi^{2} \leq 9\end{array}$ & $\begin{array}{c}\text { Best-fit } \\
\chi_{\min }^{2} \simeq 7.94 \\
\end{array}$ & $\begin{array}{c}3 \sigma \text { range } \\
\chi^{2} \leq 9\end{array}$ \\
\hline$\overline{S_{\Delta_{\mu \tau}}^{1} / 10^{-2}}$ & 2.03 & $(0.03,6.75)$ & -0.03 & $(-0.18,-0.03)$ \\
\hline$S_{\Delta_{\mu \tau}}^{2} / 10^{-2}$ & 2.10 & $(0.04,7.02)$ & -0.03 & $(-0.18,-0.03)$ \\
\hline$S_{\triangle_{\mu \tau}}^{3} / 10^{-3}$ & -2.15 & $(-7.25,-0.03)$ & 0.03 & $(0.03,0.19)$ \\
\hline$\phi_{\mu 1}^{\prime}-\phi_{\tau 1}^{\prime}$ & $1.23^{\circ}$ & $\left(-1.95^{\circ}, 4.30^{\circ}\right)$ & $-0.02^{\circ}$ & $\left(-0.19^{\circ},-0.02^{\circ}\right)$ \\
\hline$\phi_{\mu 2}^{\prime}-\phi_{\tau 2}^{\prime}$ & $2.39^{\circ}$ & $\left(0.08^{\circ}, 8.56^{\circ}\right)$ & $-0.02^{\circ}$ & $\left(-0.15^{\circ},-0.02^{\circ}\right)$ \\
\hline$\phi_{\mu 3}^{\prime}-\phi_{\tau 3}^{\prime}$ & $-3.62^{\circ}$ & $\left(-12.69^{\circ},-0.06^{\circ}\right)$ & $0.04^{\circ}$ & $\left(0.05^{\circ}, 0.32^{\circ}\right)$ \\
\hline $\mathcal{J}^{\prime} / 10^{-2}$ & -3.27 & $(-3.48,-3.03)$ & -3.32 & $(-3.39,-3.25)$ \\
\hline$m_{1}^{\prime}$ or $m_{3}^{\prime} / \mathrm{eV}$ & 0.081 & $(0,0.1)$ & $3.5 \times 10^{-5}$ & $(0,0.053)$ \\
\hline $\tan \beta$ & 24 & $(10,50)$ & 10 & $(10,24)$ \\
\hline
\end{tabular}


TABLE IV. The numerical analysis of deviations of the six LUTs at $\Lambda_{\mathrm{EW}}$ from their $\mu-\tau$ reflection symmetry limits at $\Lambda_{\mu \tau}$ for the Majorana neutrinos in the framework of the MSSM, by inputting $\left(\theta_{23}, \delta, \rho, \sigma\right)=(\pi / 4,-\pi / 2,0, \pi / 2)$ at $\Lambda_{\mu \tau}$ and allowing the smallest neutrino mass at $\Lambda_{\mathrm{EW}}\left(m_{1}^{\prime}\right.$ for the NMO case and $m_{3}^{\prime}$ for the IMO case) and the MSSM parameter $\tan \beta$ to vary in the ranges $[0,0.1] \mathrm{eV}$ and $[10,50]$, respectively.

\begin{tabular}{|c|c|c|c|c|}
\hline & \multicolumn{2}{|c|}{ Normal mass ordering (NMO) } & \multicolumn{2}{|c|}{ Inverted mass ordering (IMO) } \\
\hline & $\begin{array}{c}\text { Best-fit } \\
\chi_{\min }^{2} \simeq 0.27\end{array}$ & $\begin{array}{c}3 \sigma \text { range } \\
\chi^{2} \leq 9\end{array}$ & $\begin{array}{c}\text { Best-fit } \\
\chi_{\min }^{2} \simeq 7.94\end{array}$ & $\begin{array}{c}3 \sigma \text { range } \\
\chi^{2} \leq 9\end{array}$ \\
\hline$S_{\Delta_{\mu \tau}}^{1} / 10^{-2}$ & 6.48 & $(0.03,10.37)$ & 0.23 & $(0.22,1.33)$ \\
\hline$S_{\Delta_{\mu \tau}}^{2} / 10^{-2}$ & -1.19 & $(-4.46,0.73)$ & -0.14 & $(-0.78,-0.13)$ \\
\hline$S_{\Delta_{\mu \tau}}^{3} / 10^{-3}$ & -1.18 & $(-1.54,-0.01)$ & 0.03 & $(0.03,0.16)$ \\
\hline$\phi_{\mu 1}^{\prime}-\phi_{\tau 1}^{\prime}$ & $71.91^{\circ}$ & $\left(0.11^{\circ}, 179.99^{\circ}\right)$ & $3.16^{\circ}$ & $\left(3.05^{\circ}, 18.01^{\circ}\right)$ \\
\hline$\phi_{\mu 2}^{\prime}-\phi_{\tau 2}^{\prime}$ & $-68.10^{\circ}$ & $\left(-179.99^{\circ},-0.07^{\circ}\right)$ & $-3.08^{\circ}$ & $\left(-17.56^{\circ},-2.98^{\circ}\right)$ \\
\hline$\phi_{\mu 3}^{\prime}-\phi_{\tau 3}^{\prime}$ & $-3.81^{\circ}$ & $\left(-4.52^{\circ},-0.00001^{\circ}\right)$ & $-0.08^{\circ}$ & $\left(-0.45^{\circ},-0.06^{\circ}\right)$ \\
\hline $\mathcal{J}^{\prime} / 10^{-2}$ & -2.66 & $\left(-3.48,-8.65 \times 10^{-6}\right)$ & -3.32 & $(-3.38,-3.25)$ \\
\hline$m_{1}^{\prime}$ or $m_{3}^{\prime} / \mathrm{eV}$ & 0.030 & $(0,0.1)$ & $9.7 \times 10^{-3}$ & $(0,0.097)$ \\
\hline $\tan \beta$ & 50 & $(10,50)$ & 10 & $(10,22)$ \\
\hline
\end{tabular}

TABLE V. The numerical analysis of deviations of the six LUTs at $\Lambda_{\mathrm{EW}}$ from their $\mu-\tau$ reflection symmetry limits at $\Lambda_{\mu \tau}$ for the Majorana neutrinos in the framework of the MSSM, by inputting $\left(\theta_{23}, \delta, \rho, \sigma\right)=(\pi / 4,-\pi / 2, \pi / 2,0)$ at $\Lambda_{\mu \tau}$ and allowing the smallest neutrino mass at $\Lambda_{\mathrm{EW}}\left(m_{1}^{\prime}\right.$ for the NMO case and $m_{3}^{\prime}$ for the IMO case) and the MSSM parameter $\tan \beta$ to vary in the ranges $[0,0.1] \mathrm{eV}$ and $[10,50]$, respectively.

\begin{tabular}{|c|c|c|c|c|}
\hline & \multicolumn{2}{|c|}{ Normal mass ordering (NMO) } & \multicolumn{2}{|c|}{ Inverted mass ordering (IMO) } \\
\hline & $\begin{array}{c}\text { Best-fit } \\
\chi_{\min }^{2} \simeq 0.01\end{array}$ & $\begin{array}{c}3 \sigma \text { range } \\
\chi^{2} \leq 9\end{array}$ & $\begin{array}{c}\text { Best-fit } \\
\chi_{\min }^{2} \simeq 7.96\end{array}$ & $\begin{array}{c}3 \sigma \text { range } \\
\chi^{2} \leq 9\end{array}$ \\
\hline$\overline{S_{\Delta_{\mu \tau}}^{1} / 10^{-2}}$ & 6.59 & $(0.03,14.47)$ & 0.24 & $(0.23,1.24)$ \\
\hline$S_{\Delta_{\mu \tau}}^{2 \mu \tau} / 10^{-2}$ & 0.24 & $(-0.19,2.40)$ & -0.14 & $(-0.76,-0.14)$ \\
\hline$S_{\Delta_{\mu \tau}}^{3_{\mu \tau}^{\prime}} / 10^{-3}$ & -2.25 & $(-6.01,-0.03)$ & 0.03 & $(0.03,0.17)$ \\
\hline$\phi_{\mu 1}^{\prime}-\phi_{\tau 1}^{\prime}$ & $59.41^{\circ}$ & $\left(-2.70^{\circ}, 179.81^{\circ}\right)$ & $3.22^{\circ}$ & $\left(3.13^{\circ}, 16.94^{\circ}\right)$ \\
\hline$\phi_{\mu 2}^{\prime}-\phi_{\tau 2}^{\prime}$ & $-54.07^{\circ}$ & $\left(-179.79^{\circ}, 4.80^{\circ}\right)$ & $-3.14^{\circ}$ & $\left(-16.56^{\circ},-3.05^{\circ}\right)$ \\
\hline$\phi_{\mu 3}^{\prime}-\phi_{\tau 3}^{\prime}$ & $-5.34^{\circ}$ & $\left(-9.64^{\circ},-0.02^{\circ}\right)$ & $-0.08^{\circ}$ & $\left(-0.41^{\circ},-0.06^{\circ}\right)$ \\
\hline $\mathcal{J}^{\prime} / 10^{-2}$ & -2.85 & $(-3.48,-0.006)$ & -3.32 & $(-3.38,-3.25)$ \\
\hline$m_{1}^{\prime}$ or $m_{3}^{\prime} / \mathrm{eV}$ & 0.097 & $(0,0.1)$ & $1.3 \times 10^{-4}$ & $(0,0.062)$ \\
\hline $\tan \beta$ & 24 & $(10,50)$ & 10 & $(10,22)$ \\
\hline
\end{tabular}

or $\pi / 2$ and $\sigma=0$ or $\pi / 2$. The correspondences between the eight choices of $(\delta, \rho, \sigma)$ and the four independent cases of $\left(\eta_{1}, \eta_{2}, \eta_{3}\right)$ have been listed in Table I. Given the fact that the global-fit analysis of current neutrino oscillation data has implied a preference of $\delta$ around $-\pi / 2$ [28,29], we only focus on the case $\delta=-\pi / 2$ at $\Lambda_{\mu \tau}$ for both Dirac and Majorana neutrinos. The framework of the MSSM is typically chosen because the RGE-induced $\mu-\tau$ reflection symmetry breaking is always very small in the SM [30].

To show the deviations of the six LUTs at $\Lambda_{\mathrm{EW}}$ from their special shapes at $\Lambda_{\mu \tau}$, which can be described by the asymmetries defined in Sec. III, the numerical analysis similar to that in Ref. [15] has been done. Both the NMO $\left(m_{1}^{(\prime)}<m_{2}^{(\prime)}<m_{3}^{(\prime)}\right)$ and IMO $\left(m_{3}^{(\prime)}<m_{1}^{(\prime)}<m_{2}^{(\prime)}\right)$ cases of the Dirac or Majorana neutrinos will be taken into account.
Note that there are four choices of the two Majorana phases at $\Lambda_{\mu \tau}$, which need to be considered separately, too. In each case, we first run the relevant RGEs from $\Lambda_{\mu \tau} \sim 10^{14} \mathrm{GeV}$ down to $\Lambda_{\mathrm{EW}} \sim 10^{2} \mathrm{GeV}$ in the framework of MSSM. Here we roughly take the MSSM breaking scale $\Lambda_{\text {MSSM }}$ around $\Lambda_{\mathrm{EW}} \cdot{ }^{4}$ In the case of the Majorana neutrinos, we assume that all the heavy singlet neutrinos have a mass spectrum at

\footnotetext{
${ }^{4}$ The $\Lambda_{\text {MSSM }}$, where all superpartners are integrated out at once, is just the matching scale of SM and MSSM. It is usually assumed to be around the MSSM particle mass scales, i.e., from $1 \mathrm{TeV}$ to $10 \mathrm{TeV}$. Because the range from $\Lambda_{\mathrm{MSSM}}$ to $\Lambda_{\mathrm{EW}}$ is much smaller than the one from $\Lambda_{\mu \tau}$ to $\Lambda_{\mathrm{EW}}$ and the RGE running effect on neutrino mass parameters from $\Lambda_{\mathrm{MSSM}}$ to $\Lambda_{\mathrm{EW}}$ is very small, we can roughly take $\Lambda_{\mathrm{MSSM}} \simeq \Lambda_{\mathrm{EW}}$.
} 
TABLE VI. The numerical analysis of deviations of the six LUTs at $\Lambda_{\mathrm{EW}}$ from their $\mu-\tau$ reflection symmetry limits at $\Lambda_{\mu \tau}$ for the Majorana neutrinos in the framework of the MSSM, by inputting $\left(\theta_{23}, \delta, \rho, \sigma\right)=$ $(\pi / 4,-\pi / 2, \pi / 2, \pi / 2)$ at $\Lambda_{\mu \tau}$ and allowing the smallest neutrino mass at $\Lambda_{\mathrm{EW}}\left(m_{1}^{\prime}\right.$ for the NMO case and $m_{3}^{\prime}$ for the IMO case) and the MSSM parameter $\tan \beta$ to vary in the ranges $[0,0.1] \mathrm{eV}$ and $[10,50]$, respectively.

\begin{tabular}{|c|c|c|c|c|}
\hline & \multicolumn{2}{|c|}{ Normal mass ordering (NMO) } & \multicolumn{2}{|c|}{ Inverted mass ordering (IMO) } \\
\hline & $\begin{array}{c}\text { Best-fit } \\
\chi_{\min }^{2} \simeq 1.17\end{array}$ & $\begin{array}{c}3 \sigma \text { range } \\
\chi^{2} \leq 9\end{array}$ & $\begin{array}{c}\text { Best-fit } \\
\chi_{\min }^{2} \simeq 7.76\end{array}$ & $\begin{array}{c}3 \sigma \text { range } \\
\chi^{2} \leq 9\end{array}$ \\
\hline$S_{\Delta_{\mu \tau}}^{1} / 10^{-2}$ & 1.10 & $(0.001,1.15)$ & -0.002 & $(-0.17,-0.001)$ \\
\hline$S_{\Delta_{\mu \tau}}^{2} / 10^{-2}$ & 0.69 & $(0.001,0.74)$ & -0.002 & $(-0.18,-0.001)$ \\
\hline$S_{\Delta_{\mu \tau}}^{3} / 10^{-3}$ & -0.85 & $(-0.92,-0.001)$ & 0.002 & $(0.001,0.18)$ \\
\hline$\phi_{\mu 1}^{\prime}-\phi_{\tau 1}^{\prime}$ & $4.23^{\circ}$ & $\left(0.001^{\circ}, 4.31^{\circ}\right)$ & $-0.002^{\circ}$ & $\left(-0.15^{\circ},-0.0004^{\circ}\right)$ \\
\hline$\phi_{\mu 2}^{\prime}-\phi_{\tau 2}^{\prime}$ & $-2.67^{\circ}$ & $\left(-2.82^{\circ}, 0.13^{\circ}\right)$ & $-0.001^{\circ}$ & $\left(-0.18^{\circ},-0.001^{\circ}\right)$ \\
\hline$\phi_{\mu 3}^{\prime}-\phi_{\tau 3}^{\prime}$ & $-1.56^{\circ}$ & $\left(-1.65^{\circ},-0.002^{\circ}\right)$ & $0.003^{\circ}$ & $\left(0.003^{\circ}, 0.31^{\circ}\right)$ \\
\hline $\mathcal{J}^{\prime} / 10^{-2}$ & -3.29 & $(-3.48,-3.09)$ & -3.32 & $(-3.39,-3.24)$ \\
\hline$m_{1}^{\prime}$ or $m_{3}^{\prime} / \mathrm{eV}$ & $1.4 \times 10^{-5}$ & $(0,0.1)$ & 0.098 & $(0,0.1)$ \\
\hline $\tan \beta$ & 50 & $(10,50)$ & 10 & $(10,50)$ \\
\hline
\end{tabular}

$\Lambda_{\mu \tau}$ and are all integrated out at $\Lambda_{\mu \tau}{ }^{5}$. The initial values at $\Lambda_{\mu \tau}$ include the corresponding $\mu$ - $\tau$ reflection symmetry constraint conditions of flavor mixing angles and $C P$ phases. Furthermore, the smallest neutrino mass ( $m_{1}^{\prime}$ for the NMO case and $m_{3}^{\prime}$ for the IMO case) at $\Lambda_{\mathrm{EW}}$ and the MSSM parameter $\tan \beta$ vary in the reasonable ranges $[0,0.1] \mathrm{eV}$ and $[10,50]$, respectively. For each given values of $m_{1}^{\prime}$ or $m_{3}^{\prime}$ and $\tan \beta$, the other parameters $\left(\sin ^{2} \theta_{12}, \sin ^{2} \theta_{13}, \Delta_{\text {sol }}, \Delta_{\text {atm }}\right)$ at $\Lambda_{\mu \tau}$ are scanned over wide enough ranges by means of the MultiNest program [33], where $\Delta_{\text {sol }}=m_{2}^{2}-m_{1}^{2}$, $\Delta_{\text {atm }}=m_{3}^{2}-\left(m_{1}^{2}+m_{2}^{2}\right) / 2$, and their counterparts at $\Lambda_{\mathrm{EW}}$ $\Delta_{\text {sol }}^{\prime}=m_{2}^{\prime 2}-m_{1}^{\prime 2}$ and $\Delta_{\text {atm }}^{\prime}=m_{3}^{\prime 2}-\left(m_{1}^{\prime 2}+m_{2}^{\prime 2}\right) / 2$ have been defined to keep consistent with the notations in Ref. [28]. From each scan, we can get a set of parameters at $\Lambda_{\mathrm{EW}}$ which will be confronted with the latest global-fit results of current neutrino oscillation data by

$$
\chi^{2} \equiv \sum_{i=1}^{6} \frac{\left(\xi_{i}-\bar{\xi}_{i}\right)^{2}}{\sigma_{i}^{2}},
$$

where $\xi_{i} \in\left\{\sin ^{2} \theta_{12}^{\prime}, \sin ^{2} \theta_{13}^{\prime}, \sin ^{2} \theta_{23}^{\prime}, \delta^{\prime}, \Delta_{\text {sol }}^{\prime}, \Delta_{\text {atm }}^{\prime}\right\}$ stand for the oscillation parameters yielded from the scan; $\bar{\xi}_{i}$ and $\sigma_{i}$ denote the best-fit values and averaged $1 \sigma$ errors of $\xi_{i}$ from the global-fit analysis in Ref. [28], respectively. The best-fit values and $3 \sigma$ ranges of $\mathcal{S}_{\Delta_{\mu \tau}}^{i}, \phi_{\mu i}^{\prime}-\phi_{\tau i}^{\prime}$, and $\mathcal{J}^{\prime}$ are listed in Tables II-VI, corresponding to the

\footnotetext{
${ }^{5}$ If the heavy neutrino masses are below $\Lambda_{\mu \tau}$, we need to integrate out them successively and take into account different effective theories corresponding to different ranges of the renormalization energy scale. Thus the final results of neutrino mass parameters at $\Lambda_{\mathrm{EW}}$ running from $\Lambda_{\mu \tau}$ may be very different from our scenario under consideration [20,27,30-32].
}

minimal values $\chi_{\min }^{2}$ of $\chi^{2}$ and $\chi^{2} \leq 9$ for one degree of freedom, respectively. Considering that the two asymmetries $\mathcal{S}_{\Delta_{i}}^{\mu \tau}$ and $\phi_{\mu i}^{\prime}-\phi_{\tau i}^{\prime}$ imply consistent deviations of the LUTs, we only demonstrate the numerical results of the latter. Some discussions about the numerical results are as follows:

(i) Complementary to the analytical approximations in Sec. III, the numerical results generally reveal how the six LUTs can be reformed at $\Lambda_{\mathrm{EW}}$ by assuming the $\mu$ - $\tau$ reflection symmetry at $\Lambda_{\mu \tau}$. The reformations depend a lot on the lightest neutrino mass, the neutrino mass ordering, the Majorana phases and $\tan \beta$. From Tables II-VI, we find that the parameters running from $\Lambda_{\mu \tau}$ and their corresponding best-fit values from the global analysis in Ref. [28] can not fit very well in the IMO case, leading to big values of $\chi_{\min }^{2}$. This is mainly because the running direction of $\theta_{23}$ from $\Lambda_{\mu \tau}$ to $\Lambda_{\mathrm{EW}}$ is opposite to its best-fit value in this case $[15,18]$. The lightest neutrino mass $m_{3}^{\prime}$ and $\tan \beta$ are limited to smaller ranges by $\chi^{2} \leq 9$ in Tables III-V.

(ii) The deviations of the six LUTs are small for the case $(\rho, \sigma)=(\pi / 2, \pi / 2)$ in Table VI but their values can be very big in some other cases. For example, the two asymmetries $\phi_{\mu 1}^{\prime}-\phi_{\tau 1}^{\prime}$ and $\phi_{\mu 2}^{\prime}-$ $\phi_{\tau 2}^{\prime}$ may reach about $180^{\circ}$ in magnitude because of the smallness of the corresponding $\mathcal{J}^{\prime}$. We also notice that $\mathcal{J}^{\prime}$ running from $\Lambda_{\mu \tau}$ cannot be zero due to the nonzero value of $\mathcal{J}$ constrained by the $\mu-\tau$ reflection symmetry conditions. It is easy to understand this point from Eqs. (46) and (50).

(iii) The smallest $\chi_{\min }^{2}$ for the Dirac and Majorana neutrinos come from the best-fit results of the NMO case in Table II and Table V, respectively. The corresponding 

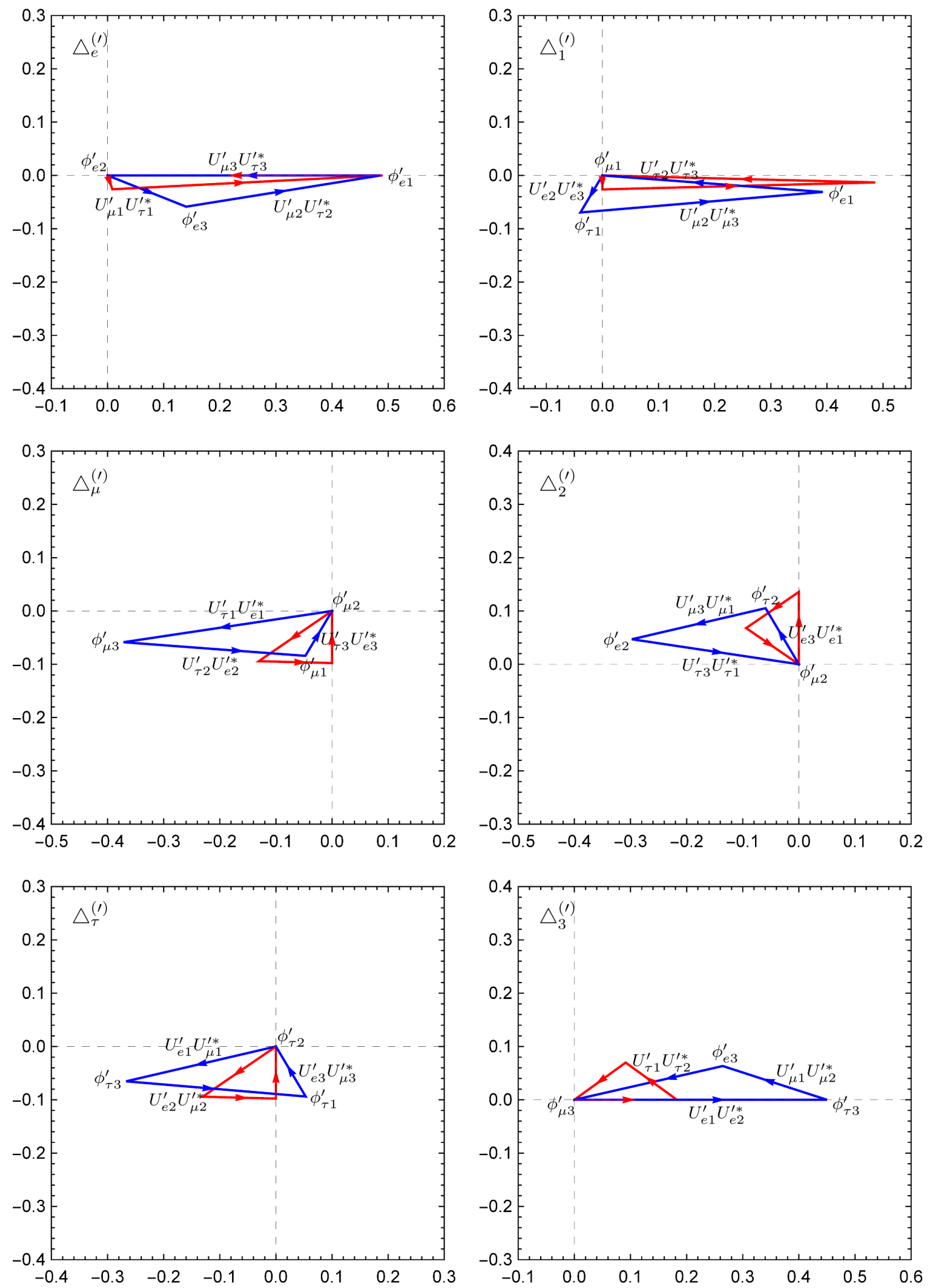

FIG. 1. The illustration of six LUTs of the Dirac neutrinos at $\Lambda_{\mathrm{EW}}$ and $\Lambda_{\mu \tau}$ in the complex plane, corresponding to the best-fit values of the NMO case in Table II $\left(\chi_{\min }^{2} \simeq 0.01\right)$, where the LUTs at $\Lambda_{\mathrm{EW}}\left(\Lambda_{\mu \tau}\right)$ are plotted in blue (red) color, and the notations of sides and inner angles belong to the blue LUTs at $\Lambda_{\mathrm{EW}}$. 

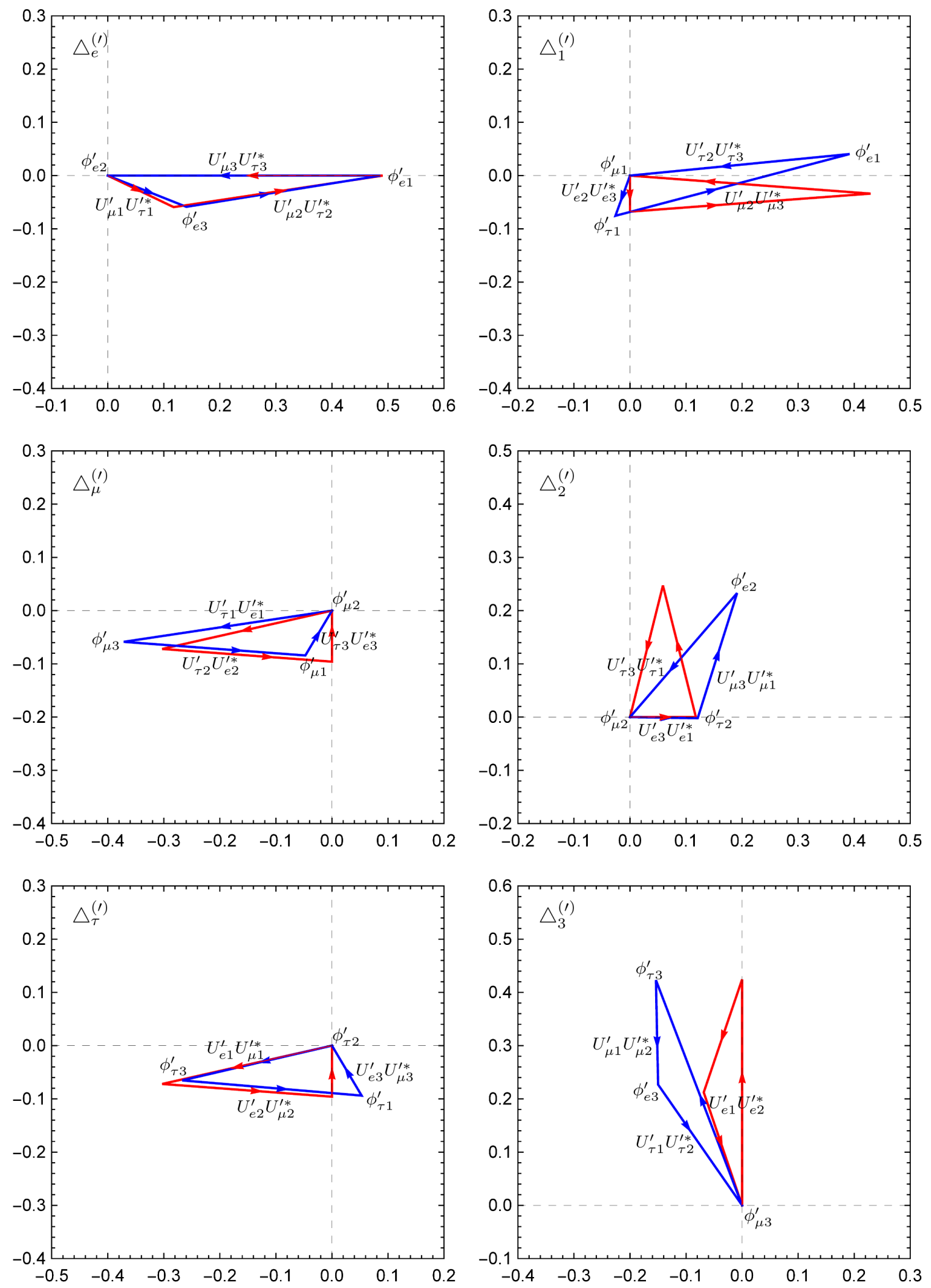

FIG. 2. The illustration of six LUTs of the Majorana neutrinos at $\Lambda_{\mathrm{EW}}$ and $\Lambda_{\mu \tau}$ in the complex plane, corresponding to the best-fit values of the NMO case in Table $\mathrm{V}\left(\chi_{\min }^{2} \simeq 0.01\right)$, where the LUTs at $\Lambda_{\mathrm{EW}}\left(\Lambda_{\mu \tau}\right)$ are plotted in blue (red) color, and the notations of sides and inner angles belong to the blue LUTs at $\Lambda_{\mathrm{EW}}$. 
LUTs together with their counterparts at $\Lambda_{\mu \tau}$ have been specifically shown in Figs. 1 and 2. The blue triangles with $\chi_{\min }^{2} \simeq 0.01$ stand for the LUTs at $\Lambda_{\mathrm{EW}}$ and almost overlap the LUTs implied by the best-fit values of the global analysis in Ref. [28], while the red ones denote the corresponding LUTs at $\Lambda_{\mu \tau}$. When comparing the two figures, we find that the blue LUTs at $\Lambda_{\mathrm{EW}}$ differ with each other only in the orientations of $\Delta_{i}$ caused by the Majorana phases, while the red ones are very different.

\section{SUMMARY}

The neutrino physics has promisingly entered the era of precision measurements, providing us more information to understand the large-angle lepton favor mixing pattern and potentially big $C P$-violating phases. From the perspective of model construction, we usually introduce heavy degrees of freedom and flavor symmetry at a superhigh energy scale to explain the smallness of neutrino masses and the observed results of neutrino oscillation parameters at $\Lambda_{\mathrm{EW}}$. In this paper, we use the LUTs to describe the RGE running effects of lepton flavor mixing. The analytical results in the integral form can directly connect two LUTs at $\Lambda_{\mathrm{H}}$ and $\Lambda_{\mathrm{EW}}$, and they complement to the corresponding results of the differential form in Ref. [12]. We also apply the LUT language to the description of the $\mu-\tau$ reflection symmetry, whose RGE-induced breaking effects can be intuitively interpreted as the deviations of the LUTs from their special shapes at $\Lambda_{\mu \tau}$. The reformations of the six LUTs from $\Lambda_{\mu \tau}$ to $\Lambda_{\mathrm{EW}}$ have been analytically and numerically studied in a general way. Their dependence on the lightest neutrino mass, neutrino mass ordering, Majorana phases and the MSSM parameter $\tan \beta$ have been revealed, corresponding to the dependence of specific flavor mixing parameters on these factors $[14,15,18,24,26,27]$. We hope this work can enrich the neutrino phenomenology and help to understand the relevant underlying physics.

\section{ACKNOWLEDGMENTS}

I would like to thank Prof. Zhi-zhong Xing for suggesting that I study this topic, many useful discussions, and reading the manuscript. I am also grateful to Di Zhang and Guo-yuan Huang for many useful discussions. This work was supported in part by the National Natural Science Foundation of China under Grant No. 11775231.

\section{APPENDIX: THE EXACT EXPRESSIONS OF $m_{i}^{\prime 2}$}

By solving Eq. (11), we get the exact expressions of Dirac neutrino mass squares $m_{i}^{\prime 2}$ at $\Lambda_{\mathrm{EW}}$ and write them as

$$
\begin{aligned}
& m_{1}^{\prime 2}=\frac{x}{3}-\frac{\sqrt{x^{2}-3 y}}{3}\left[z+\sqrt{3\left(1-z^{2}\right)}\right] \\
& m_{2}^{\prime 2}=\frac{x}{3}-\frac{\sqrt{x^{2}-3 y}}{3}\left[z-\sqrt{3\left(1-z^{2}\right)}\right] \\
& m_{3}^{\prime 2}=\frac{x}{3}+\frac{2 z \sqrt{x^{2}-3 y}}{3}
\end{aligned}
$$

where $x=b, y=\left(b^{2}-c\right) / 2$ and

$$
z=\cos \left[\frac{1}{3} \arccos \frac{2 x^{3}-9 x y+27 a}{2\left(x^{2}-3 y\right)^{3 / 2}}\right] .
$$

The Majorana neutrino mass squares $m_{i}^{\prime 2}$ at $\Lambda_{\mathrm{EW}}$ can also be exactly shown as the same form of Eq. (A1) by replacing the definitions of $(a, b, c)$ with those defined below Eq. (29). Note that Eq. (A1) applies to the NMO case and in the IMO case, we need to do the replacements $m_{1}^{\prime 2} \rightarrow m_{3}^{\prime 2}, m_{2}^{\prime 2} \rightarrow m_{1}^{\prime 2}$ and $m_{3}^{\prime 2} \rightarrow m_{2}^{\prime 2}$. One can find that the three Dirac or Majorana neutrino mass squares $m_{i}^{\prime 2}$ at $\Lambda_{\mathrm{EW}}$ running from $\Lambda_{\mathrm{H}}$ look similar to the effective neutrino mass squares in constant density matter [34] except the different expressions of $x, y$ and $z$ therein.
[1] M. Tanabashi et al. (Particle Data Group), Phys. Rev. D 98, 030001 (2018).

[2] Z. Maki, M. Nakagawa, and S. Sakata, Prog. Theor. Phys. 28, 870 (1962).

[3] B. Pontecorvo, Zh. Eksp. Teor. Fiz. 53, 1717 (1967) [Sov. Phys. JETP 26, 984 (1968)].

[4] H. Fritzsch and Z.z. Xing, Prog. Part. Nucl. Phys. 45, 1 (2000).

[5] J. A. Aguilar-Saavedra and G. C. Branco, Phys. Rev. D 62, 096009 (2000).
[6] J. Sato, Nucl. Instrum. Methods Phys. Res., Sect. A 472, 434 (2001); Y. Farzan and A. Yu. Smirnov, Phys. Rev. D 65, 113001 (2002).

[7] Y. Koide, Phys. Rev. D 73, 073002 (2006); J. D. Bjorken, P. F. Harrison, and W. G. Scott, Phys. Rev. D 74, 073012 (2006); G. Ahuja and M. Gupta, Phys. Rev. D 77, 057301 (2008); A. Dueck, S. Petcov, and W. Rodejohann, Phys. Rev. D 82, 013005 (2010).

[8] S. Antusch, C. Biggio, E. Fernandez-Martinez, M. B. Gavela, and J. Lopez-Pavon, J. High Energy Phys. 10 (2006) 084. 
[9] H. J. He and X. J. Xu, Phys. Rev. D 89, 073002 (2014); 95, 033002 (2017).

[10] Z.z. Xing and J. y. Zhu, Nucl. Phys. B908, 302 (2016).

[11] Z. z. Xing, Int. J. Mod. Phys. A 19, 1 (2004); H. Zhang and Z. z. Xing, Eur. Phys. J. C 41, 143 (2005); Z. z. Xing and H. Zhang, Phys. Lett. B 618, 131 (2005); Z. z. Xing and J. y. Zhu, J. High Energy Phys. 07 (2016) 011.

[12] S. Luo, Phys. Rev. D 85, 013006 (2012).

[13] P. F. Harrison and W. G. Scott, Phys. Lett. B 547, 219 (2002).

[14] Z. z. Xing and Z.h. Zhao, Rep. Prog. Phys. 79, 076201 (2016); and references therein.

[15] G. y. Huang, Z. z. Xing, and J. y. Zhu, Chin. Phys. C 42, 123108 (2018).

[16] W. H. Furry, Phys. Rev. 56, 1184 (1939); S. M. Bilenky and C. Giunti, Int. J. Mod. Phys. A 30, 1530001 (2015); S. Dell'Oro, S. Marcocci, M. Viel, and F. Vissani, Adv. High Energy Phys. 2016, 2162659 (2016).

[17] R. N. Mohapatra and J. W. F. Valle, Phys. Rev. D 34, 1642 (1986); N. Arkani-Hamed, L. J. Hall, H. Murayama, D. Tucker-Smith, and N. Weiner, Phys. Rev. D 64, 115011 (2001); F. Borzumati and Y. Nomura, Phys. Rev. D 64, 053005 (2001); R. Kitano, Phys. Lett. B 539, 102 (2002); S. Abel, A. Dedes, and K. Tamvakis, Phys. Rev. D 71, 033003 (2005).

[18] Z. z. Xing, D. Zhang, and J. y. Zhu, J. High Energy Phys. 11 (2017) 135.

[19] G. C. Branco, P. M. Ferreira, L. Lavoura, M. N. Rebelo, M. Sher, and J. P. Silva, Phys. Rep. 516, 1 (2012).

[20] S. Antusch, J. Kersten, M. Lindner, M. Ratz, and M. A. Schmidt, J. High Energy Phys. 03 (2005) 024.

[21] P. Minkowski, Phys. Lett. B 67, 421 (1977); T. Yanagida, in Proceedings of the Workshop on Unified Theory and the Baryon Number of the Universe, edited by O. Sawada and A. Sugamoto (KEK, Tsukuba, 1979), p. 95; M. Gell-Mann, P. Ramond, and R. Slansky, in Supergravity, edited by P. van Nieuwenhuizen and D. Freedman (North Holland, Amsterdam, 1979), p. 315; S. L. Glashow, in Quarks and Leptons, edited by M. Lévy et al. (Plenum,
New York, 1980), p. 707; R. N. Mohapatra and G. Senjanovic, Phys. Rev. Lett. 44, 912 (1980); For a brief review of other seesaw mechanisms, see: Z. Z. Xing, Prog. Theor. Phys. Suppl. 180, 112 (2009).

[22] J. R. Ellis and S. Lola, Phys. Lett. B 458, 310 (1999).

[23] Z. z. Xing, Phys. Lett. B 633, 550 (2006).

[24] Y. L. Zhou, arXiv:1409.8600.

[25] N. Aghanim et al. (Planck Collaboration), arXiv:1807.06209.

[26] S. Luo and Z.z. Xing, Phys. Rev. D 90, 073005 (2014); Z.h. Zhao, J. High Energy Phys. 09 (2017) 023; W. Rodejohann and X.J. Xu, Phys. Rev. D 96, 055039 (2017); Z. C. Liu, C. X. Yue, and Z. h. Zhao, J. High Energy Phys. 10 (2017) 102.

[27] N. Nath, Z. z. Xing, and J. Zhang, Eur. Phys. J. C 78, 289 (2018).

[28] F. Capozzi, E. Lisi, A. Marrone, and A. Palazzo, Prog. Part. Nucl. Phys. 102, 48 (2018).

[29] P. F. de Salas, D. V. Forero, C. A. Ternes, M. Tortola, and J. W. F. Valle, Phys. Lett. B 782, 633 (2018); I. Esteban, M. C. Gonzalez-Garcia, M. Maltoni, I. Martinez-Soler, and T. Schwetz, J. High Energy Phys. 01 (2017) 087; and NuFIT 3.2 (2018), www.nu-fit.org.

[30] T. Ohlsson and S. Zhou, Nat. Commun. 5, 5153 (2014).

[31] S. Antusch, J. Kersten, M. Lindner, and M. Ratz, Phys. Lett. B 538, 87 (2002).

[32] J. w. Mei and Z. z. Xing, Phys. Rev. D 70, 053002 (2004); J. w. Mei, Phys. Rev. D 71, 073012 (2005); S. Gupta, S. K. Kang, and C. S. Kim, Nucl. Phys. B893, 89 (2015); J. Zhang and S. Zhou, J. High Energy Phys. 09 (2016) 167; S. F. King, J. Zhang, and S. Zhou, J. High Energy Phys. 12 (2016) 023.

[33] F. Feroz and M. P. Hobson, Mon. Not. R. Astron. Soc. 384, 449 (2008); F. Feroz, M. P. Hobson, M. Bridges, F. Feroz, M. P. Hobson, E. Cameron, and A. N. Pettitt, arXiv:1306 .2144 .

[34] V. D. Barger, K. Whisnant, S. Pakvasa, and R. J. N. Phillips, Phys. Rev. D 22, 2718 (1980); H. W. Zaglauer and K. H. Schwarzer, Z. Phys. C 40, 273 (1988). 